\title{
How to control pairing fluctuations: SU(2) slave-rotor gauge theory of the Hubbard model
}

\author{
Ki-Seok Kim \\ School of Physics, Korea Institute for Advanced Study, Seoul 130-012, Korea
}

(Dated: September 7, 2018)

\begin{abstract}
We study how to incorporate Mott physics in the BCS-type superconductor, motivated from the fact that high $T_{c}$ superconductivity results from a Mott insulator via hole doping. The U(1) slaverotor representation was proposed to take local density fluctuations into account non-perturbatively, describing the Mott-Hubbard transition at half filling. Since this decomposition cannot control local pairing fluctuations, the U(1) slave-rotor representation does not give a satisfactory treatment for charge fluctuations. Extending the U(1) slave-rotor representation, we introduce an $\mathrm{SU}(2)$ slaverotor representation to allow not only local density fluctuations but also local pairing excitations. We find an SU(2) slave-rotor gauge theory of the Hubbard model in terms of two kinds of collective boson excitations associated with density and pairing fluctuations that interact with gapless fermion excitations via $\mathrm{SU}(2)$ gauge fluctuations. An interesting observation in this effective description is that phase fluctuations of fermion pairs arise as $\mathrm{SU}(2)$ gauge fluctuations. Thus, fermion-pairing excitations can be controlled by dynamics of collective bosons in the $\mathrm{SU}(2)$ slave-rotor gauge theory. Performing the standard saddle-point analysis based on the $\mathrm{SU}(2)$ slave-rotor action, we find an interesting phase described by partial freezing of charge fluctuations near the Mott-Hubbard critical point, where local density-fluctuation modes are condensed but local pair-excitation modes are gapped. Partial freezing of charge fluctuations causes fermion pairing to be incoherent as a result of reconciliation of superconductivity and Mott physics. The nature of this non-superconducting phase is identified with an anomalous metal due to the presence of incoherent pairing.
\end{abstract}

PACS numbers: 71.10.Hf, 71.30.+h, 71.10.-w, 71.10.Fd

\section{INTRODUCTION}

Nature of the doped Mott insulator is one of the central interests in modern condensed matter physics, associated with the mechanism of high $T_{c}$ superconductivity. Considering the high $T_{c}$ phase diagram, an antiferromagnetic order in the parent Mott insulator vanishes rapidly via hole doping, and a paramagnetic non-superconducting phase appears. This non-magnetic state evolves into the high $T_{c}$ superconductor, doping holes further.[1] The central issue is the nature of the intermediate non-magnetic phase between the antiferromagnetic Mott insulator and high $T_{c}$ superconductor. It is important to notice that physics of the superconducting state is BCS-like although the high $T_{c}$ superconductivity results from the doped Mott insulator.[1] In this respect the intermediate phase will be determined by competition of Mott physics and BCS superconductivity. In this paper we discuss how to incorporate the Mott physics into the BCS superconductivity.

To understand the Mott physics in a concrete manner, we consider the BCS-Hubbard Hamiltonian

$$
\begin{aligned}
& H=-t \sum_{\langle i j\rangle \sigma}\left(c_{i \sigma}^{\dagger} c_{j \sigma}+H . c .\right) \\
& -\sum_{\langle i j\rangle}\left|\Delta_{i j}\right| e^{-i a_{i j}}\left(c_{i \uparrow}^{\dagger} c_{j \downarrow}^{\dagger}-c_{i \downarrow}^{\dagger} c_{j \uparrow}^{\dagger}\right)-H . c .+\frac{1}{J} \sum_{\langle i j\rangle}\left|\Delta_{i j}\right|^{2} \\
& +U \sum_{i} n_{i \uparrow} n_{i \downarrow},
\end{aligned}
$$

where $\Delta_{i j}$ is the pairing potential with its amplitude
$\left|\Delta_{i j}\right|$ and phase $a_{i j}$. This Hamiltonian introduces the competing nature arising from the density-phase uncertainty; the $J$ term causes local pairing of electrons while the Hubbard- $U$ term suppresses local charge fluctuations, thus breaking phase coherence of electron pairs. When local charge fluctuations are strong in the case of $U<U_{c}$ with the critical strength $U_{c}$ for the Mott transition, phase fluctuations of electron pairs would be suppressed, i.e., $\left\langle e^{-i a_{i j}}\right\rangle=1$ owing to the densityphase uncertainty relation. In this case superconductivity is expected to appear, described by the BCS model $L_{S C}=\sum_{i \sigma} c_{i \sigma}^{\dagger}\left(\partial_{\tau}-\mu\right) c_{i \sigma}-t \sum_{\langle i j\rangle \sigma}\left(c_{i \sigma}^{\dagger} c_{j \sigma}+H . c.\right)-$ $\sum_{\langle i j\rangle}\left|\Delta_{i j}\right|\left(c_{i \uparrow}^{\dagger} c_{j \downarrow}^{\dagger}-c_{i \downarrow}^{\dagger} c_{j \uparrow}^{\dagger}\right)-$ H.c. On the other hand, when local density fluctuations are suppressed in the case of $U>U_{c}$, the density-phase uncertainty causes $\left\langle e^{-i a_{i j}}\right\rangle=$ 0 . The resulting non-superconducting phase would be described by the effective Lagrangian $L_{e f f}=\sum_{i \sigma} c_{i \sigma}^{\dagger}\left(\partial_{\tau}-\right.$ $\mu) c_{i \sigma}-t \sum_{\langle i j\rangle \sigma}\left(c_{i \sigma}^{\dagger} c_{j \sigma}+\right.$ H.c. $)-\sum_{\langle i j\rangle}\left|\Delta_{i j}\right| e^{-i a_{i j}}\left(c_{i \uparrow}^{\dagger} c_{j \downarrow}^{\dagger}-\right.$ $\left.c_{i \downarrow}^{\dagger} c_{j \uparrow}^{\dagger}\right)-H . c .-\frac{1}{g^{2}} \sum_{\square} \cos (\partial \times a)$, where the gauge action is introduced to impose $\left\langle e^{-i a_{i j}}\right\rangle=0$. This state can be regarded as a non-Fermi liquid metal with phaseincoherent pairs. $[2,3]$

The problem is how to control local charge fluctuations. Recently, U(1) slave-rotor representation was proposed in order to take local charge fluctuations into account non-perturbatively.[4] Actually, the U(1) slaverotor gauge theory of the Hubbard Hamiltonian found the bandwidth-control Mott transition from spin liquid to Fermi liquid at half filling, ignoring antiferromagnetism and superconductivity.[4-6] Using the U(1) slave-rotor 
decomposition $c_{i \sigma}=e^{-i \theta_{i}} f_{i \sigma}$, we obtain the following expression from Eq. (1)

$$
\begin{aligned}
& Z=\int D f_{i \sigma} D \theta_{i} D\left|\Delta_{i j}\right| D a_{i j} D L_{i} D \varphi_{i} e^{-\int_{0}^{\beta} d \tau L}, \\
& L=\sum_{i \sigma} f_{i \sigma}^{\dagger}\left(\partial_{\tau}-\mu\right) f_{i \sigma}-t \sum_{\langle i j\rangle \sigma}\left(f_{i \sigma}^{\dagger} e^{i \theta_{i}} e^{-i \theta_{j}} f_{j \sigma}+H . c .\right) \\
& +\sum_{i}\left(\frac{U}{4} L_{i}^{2}-i L_{i} \partial_{\tau} \theta_{i}+i \varphi_{i}\left(L_{i}-\left[\sum_{\sigma} f_{i \sigma}^{\dagger} f_{i \sigma}-1\right]\right)\right) \\
& -\sum_{\langle i j\rangle}\left|\Delta_{i j}\right| e^{i \theta_{i}} e^{-i a_{i j}} e^{i \theta_{j}}\left(f_{i \uparrow}^{\dagger} f_{j \downarrow}^{\dagger}-f_{i \downarrow}^{\dagger} f_{j \uparrow}^{\dagger}\right)-H . c . \\
& +\frac{1}{J} \sum_{\langle i j\rangle}\left|\Delta_{i j}\right|^{2},
\end{aligned}
$$

where the Hubbard- $U$ term is represented as the charge and spin channels $U \sum_{i} n_{i \uparrow} n_{i \downarrow}=\frac{U}{4} \sum_{i}\left(n_{i \uparrow}+n_{i \downarrow}-1\right)^{2}-$ $\frac{U}{4} \sum_{i}\left(n_{i \uparrow}-n_{i \downarrow}\right)^{2}+\frac{U}{2} \sum_{i}\left(n_{i \uparrow}+n_{i \downarrow}\right)-\frac{U}{4} \sum_{i} 1$, and the spin channel is not taken into account in this paper. It is easy to show that Eq. (2) is exactly the same as Eq. (1) with the charge channel for the Hubbard- $U$ term, integrating out the $\varphi_{i}$ and $L_{i}$ fields with $f_{i \sigma}=e^{i \theta_{i}} c_{i \sigma}$. In this expression the electron Hilbert space $\mid c_{i \sigma}>$ is reconstructed as the direct product of the boson and fermion Hilbert spaces $\left|L_{i}>\otimes\right| f_{i \sigma}>$ according to the decomposition $c_{i \sigma}=e^{-i \theta_{i}} f_{i \sigma}$, where $L_{i}$ represents an electron density at site $i$. It is clear that any decomposition method enlarges the original electron Hilbert space, thus an appropriate constraint associated with the decomposition should be imposed. The Lagrange multiplier field $\varphi_{i}$ expresses the $\mathrm{U}(1)$ slave-rotor constraint $L_{i}=\sum_{\sigma} f_{i \sigma}^{\dagger} f_{i \sigma}-1$, implying that the fermion and boson Hilbert spaces are not independent, thus the two operators $f_{i \sigma}$ and $e^{i \theta_{i}}$ also. Then, $e^{-i \theta_{i}}$ is identified with an annihilation operator of an electron charge owing to the constraint $L_{i}=\sum_{\sigma} f_{i \sigma}^{\dagger} f_{i \sigma}-1$ and the canonical relation $\left[L_{i}, \theta_{j}\right]=-i \delta_{i j}$ imposed by $-i L_{i} \partial_{\tau} \theta_{i}$.

Integrating out the density field $L_{i}$ and performing the Hubbard-Stratonovich (HS) transformation to decompose the "correlated" hopping term in the following way

$$
\begin{aligned}
& \exp \left[-\int_{0}^{\beta} d \tau\left\{-t \sum_{\langle i j\rangle \sigma}\left(f_{i \sigma}^{\dagger} e^{i \theta_{i}} e^{-i \theta_{j}} f_{j \sigma}+H . c .\right)\right\}\right] \\
& =\int D \alpha_{i j} D \beta_{i j} \exp \left[-\int_{0}^{\beta} d \tau\left\{t \sum_{\langle i j\rangle}\left(\alpha_{i j}^{*} \beta_{i j}+H . c .\right)\right.\right. \\
& \left.\left.-t \sum_{\langle i j\rangle \sigma}\left(f_{i \sigma}^{\dagger} \alpha_{i j}^{*} f_{j \sigma}+H . c .\right)-t \sum_{\langle i j\rangle}\left(e^{i \theta_{i}} \beta_{i j} e^{-i \theta_{j}}+H . c .\right)\right\}\right]
\end{aligned}
$$

we obtain the $\mathrm{U}(1)$ slave-rotor gauge Lagrangian with $d-$ wave pairing

$$
\begin{aligned}
& Z=\int D f_{i \sigma} D \theta_{i} D a_{i j} D \varphi_{i} D c_{i j} e^{-\int_{0}^{\beta} d \tau L}, \\
& L=L_{0}+L_{f}+L_{\theta}, \quad L_{0}=\sum_{\langle i j\rangle}\left(t \alpha \beta+\frac{\Delta^{2}}{J}\right), \\
& L_{f}=\sum_{i \sigma} f_{i \sigma}^{\dagger}\left(\partial_{\tau}-\mu\right) f_{i \sigma}-i \sum_{i} \varphi_{i}\left[\sum_{\sigma} f_{i \sigma}^{\dagger} f_{i \sigma}-1\right] \\
& -t \alpha \sum_{\langle i j\rangle \sigma}\left(f_{i \sigma}^{\dagger} e^{-i c_{i j}} f_{j \sigma}+H . c .\right) \\
& -\Delta \sum_{\langle i j\rangle} \varsigma_{i j} e^{-i a_{i j}}\left(f_{i \uparrow}^{\dagger} f_{j \downarrow}^{\dagger}-f_{i \downarrow}^{\dagger} f_{j \uparrow}^{\dagger}\right)-H . c ., \\
& L_{\theta}=\frac{1}{U} \sum_{i}\left(\partial_{\tau} \theta_{i}-\varphi_{i}\right)^{2}-t \beta \sum_{\langle i j\rangle}\left(e^{i \theta_{i}} e^{i c_{i j}} e^{-i \theta_{j}}+H . c .\right),
\end{aligned}
$$

where the hopping parameters are represented as $\alpha_{i j}=$ $\alpha e^{i c_{i j}}$ and $\beta_{i j}=\beta e^{i c_{i j}}$ with amplitudes $\alpha, \beta$ and phase $c_{i j}$, and $d$-wave pairing-symmetry is assumed in $\varsigma_{i j}$ of $\left|\Delta_{i j}\right|=\Delta \varsigma_{i j}$ with the gauge transformation $a_{i j} \rightarrow a_{i j}+$ $\theta_{i}+\theta_{j}$. The unidentified hopping and pairing amplitudes can be determined self-consistently in the saddle-point analysis, $\alpha=\left|\left\langle e^{-i \theta_{i}} e^{i \theta_{j}}+H . c.\right\rangle\right|, \beta=\left|\left\langle\sum_{\sigma} f_{i \sigma}^{\dagger} f_{j \sigma}+H . c.\right\rangle\right|$ and $\Delta s_{i j}=\left|\left\langle f_{i \uparrow}^{\dagger} f_{j \downarrow}^{\dagger}-f_{i \downarrow}^{\dagger} f_{j \uparrow}^{\dagger}\right\rangle\right|$.

This effective Lagrangian is quite appealing. Starting from the BCS-Hubbard effective model, we extract dynamics of collective charge fluctuations from the Hubbard- $U$ term. As a result, the fermion sector corresponds to the BCS description with a renormalized bandwidth $D \alpha$ while dynamics of collective density excitations is described by the boson-Hubbard-type model with an effective chemical potential in the saddle-point approximation $\left(c_{i j}=0\right)$. Remember that the boson-Hubbard model is the prototype for the quantum phase transition associated with charge fluctuations, describing the genuine Mott transition without symmetry breaking via condensation. The quantum transition indeed occurs when $D \beta / U \sim 1$ with the half bandwidth $D$, where $D \beta$ is an effective bandwidth for the boson field. One can show that the hopping parameter $\beta$ decreases as $U$ increases, resulting in the bandwidth-control Mott-Hubbard transition. Actually, the previous slave-rotor studies showed this transition without superconductivity $(\Delta=0)$ at half filling. [4-6]

However, this treatment does not take into account phase fluctuations $e^{-i a_{i j}}$ of fermion pairs carefully although local density fluctuations are governed by U(1) rotor excitations $e^{i \theta_{i}}$. In other words, the U(1) slaverotor representation cannot give any condition for pairing excitations. Considering the mathematical structure of Eq. (3), one can find two kinds of gauge excitations, $c_{i j}$ and $a_{i j}$ associated with local density fluctuations and pairing excitations. Density-gauge excitations $\left(c_{i j}\right)$ can be controlled by $\mathrm{U}(1)$ rotor excitations $e^{i \theta_{i}}$. When local density fluctuations are suppressed in the large $U$ limit, 
boson excitations are gapped (Mott insulator), causing gapless density-gauge fluctuations. On the other hand, when local density fluctuations become strong in the small $U$ limit, the $\mathrm{U}(1)$ rotor variables get condensed, making density-gauge fluctuations massive due to the Anderson-Higgs mechanism. However, there are no such boson excitations corresponding to the $\mathrm{U}(1)$ density-rotor variable for pair-gauge fluctuations $\left(a_{i j}\right)$.

This discussion motivates us to introduce new boson excitations associated with local pairing fluctuations, allowing us to control phase fluctuations of pairing excitations (pair-gauge fluctuations). Assume the presence of such boson excitations. We can estimate that both density and pairing fluctuations would be gapped due to the density-phase uncertainty in the large $U$ limit. On the other hand, both collective excitations become condensed in the small $U$ limit. What happens near the Mott critical point? It should be noted that local pair excitations are different from local density fluctuations. Thus, there is no reason for both excitations to be condensed at the same time. Can there exist an intermediate phase, where only one kind of boson excitations is condensed. If possible, what is the nature of this intermediate phase?

In this paper we extend the $\mathrm{U}(1)$ slave-rotor formulation, allowing local pairing fluctuations. Then, the collective boson field is expressed as an $\mathrm{SU}(2)$ matrix field $U_{i \sigma \sigma^{\prime}}=\left(\begin{array}{cc}z_{i \uparrow} & -z_{i \downarrow}^{\dagger} \\ z_{i \downarrow} & z_{i \uparrow}^{\dagger}\end{array}\right)$, involved with both density $\left(z_{i \uparrow}\right)$ and pairing $\left(z_{i \downarrow}\right)$ fluctuations.[7] This leads us to construct an $\mathrm{SU}(2)$ slave-rotor gauge theory of the Hubbard model in terms of the collective density- and pairingfluctuation modes interacting with gapless fermion excitations via $\mathrm{SU}(2)$ gauge fluctuations. [8] Compared with the $\mathrm{U}(1)$ slave-rotor gauge theory [Eq. (3)], $z_{i \uparrow}$ corresponds to $e^{i \theta_{i}}$ roughly speaking while $z_{i \downarrow}$ is newly introduced to control pairing-gauge fluctuations (phase fluctuations of electron pairs). Since the SU(2) slave-rotor gauge theory admits two kinds of collective bosons, an intermediate phase can be naturally allowed between the spin liquid Mott insulator $\left(\left\langle z_{i \uparrow}\right\rangle=0,\left\langle z_{i \downarrow}\right\rangle=0\right)$ and Fermi liquid metal $\left(\left\langle z_{i \uparrow}\right\rangle \neq 0,\left\langle z_{i \downarrow}\right\rangle \neq 0\right)$, characterized by softening of the density-fluctuation modes $\left(\left\langle z_{i \uparrow}\right\rangle \neq 0\right)$ as the Fermi liquid, but gapping of the pair-excitation modes $\left(\left\langle z_{i \downarrow}\right\rangle=0\right)$ as the spin liquid. Remember that the $\mathrm{U}(1)$ slave-rotor gauge theory contains only the densityfluctuation modes $\left(z_{i \uparrow}\right)$, thus allowing the two phases only, the spin liquid Mott insulator $\left(\left\langle z_{i \uparrow}\right\rangle=0\right)$ and Fermi liquid metal $\left(\left\langle z_{i \uparrow}\right\rangle \neq 0\right)$.

Performing a mean-field analysis based on an SU(2) slave-rotor effective action, we find the intermediate phase indeed away from half filling, described by partial freezing of charge fluctuations $\left(\left\langle z_{i \uparrow}\right\rangle \neq 0,\left\langle z_{i \downarrow}\right\rangle=0\right)$ near the Mott-Hubbard critical point. We reveal that this intermediate phase originates from emergence of a pseudospin-dependent chemical potential due to hole doping that causes $\mathrm{SU}(2)$ pseudospin symmetry breaking to discriminate the local pair-excitation modes $\left(z_{i \downarrow}\right)$ from the local density-fluctuation modes $\left(z_{i \uparrow}\right)$. Condensation of density-fluctuation modes makes density-gauge excitations gapped due to the Anderson-Higgs mechanism. On the other hand, pairing-gauge fluctuations remain gapless because pairing-excitation modes are gapped. As a result, we find an effective $\mathrm{U}(1)$ gauge theory for this intermediate phase, where phase fluctuations of fermion pairs are described by pairing-gauge fluctuations. We discuss physics of this non-superconducting phase, identified with a non-Fermi liquid metal due to the presence of phase-incoherent pairs.

\section{SU(2) SLAVE-ROTOR THEORY OF THE HUBBARD MODEL}

\section{A. SU(2) slave-rotor representation in the path integral formulation}

We start from the Hubbard Hamiltonian

$$
H=-t \sum_{\langle i j\rangle \sigma}\left(c_{i \sigma}^{\dagger} c_{j \sigma}+H . c .\right)+\frac{3 u}{2} \sum_{i} c_{i \uparrow}^{\dagger} c_{i \uparrow} c_{i \downarrow}^{\dagger} c_{i \downarrow},(4)
$$

where $u / t$ is a parameter determining various phases of this model with hole concentration. The local interaction term can be decomposed into the pairing and density channels in the following way

$$
\begin{aligned}
& \frac{3 u}{2} \sum_{i} c_{i \uparrow}^{\dagger} c_{i \uparrow} c_{i \downarrow}^{\dagger} c_{i \downarrow}=\frac{u}{2} \sum_{i} c_{i \uparrow}^{\dagger} c_{i \downarrow}^{\dagger} c_{i \downarrow} c_{i \uparrow} \\
& +\frac{u}{2} \sum_{i}\left(\sum_{\sigma} c_{i \sigma}^{\dagger} c_{i \sigma}-1\right)^{2}+\frac{u}{2}\left(\sum_{\sigma} c_{i \sigma}^{\dagger} c_{i \sigma}-1\right) .
\end{aligned}
$$

Performing the HS transformation for the pairing- and density-interaction channels, we find an effective Lagrangian in the Nambu-spinor representation

$$
\begin{aligned}
& Z=\int D\left[\psi_{i}, \Phi_{i}^{R}, \Phi_{i}^{I}, \varphi_{i}\right] e^{-\int_{0}^{\beta} d \tau L} \\
& L=\sum_{i} \psi_{i}^{\dagger}\left(\partial_{\tau} \mathbf{I}-\mu \tau_{3}\right) \psi_{i}-t \sum_{\langle i j\rangle}\left(\psi_{i}^{\dagger} \tau_{3} \psi_{j}+H . c .\right) \\
& -i \sum_{i}\left(\Phi_{i}^{R} \psi_{i}^{\dagger} \tau_{1} \psi_{i}+\Phi_{i}^{I} \psi_{i}^{\dagger} \tau_{2} \psi_{i}+\varphi_{i} \psi_{i}^{\dagger} \tau_{3} \psi_{i}\right) \\
& +\frac{1}{2 u} \sum_{i}\left(\Phi_{i}^{R 2}+\Phi_{i}^{I 2}+\varphi_{i}^{2}\right)
\end{aligned}
$$

Here $\psi_{i}$ is the Nambu spinor, given by $\psi_{i}=\left(\begin{array}{c}c_{i \uparrow} \\ c_{i \downarrow}^{\dagger}\end{array}\right) . \Phi_{i}^{R}$ and $\Phi_{i}^{I}$ are the real and imaginary parts of the on-site pairing-order parameter respectively, and $\varphi_{i}$ is an effective density-potential. $\mu$ is an electron chemical potential, renormalized by local interactions as $\mu=\mu_{b}-u / 2$, where $\mu_{b}$ is the bare chemical potential. Introducing a pseudospin vector $\vec{\Omega}_{i} \equiv\left(\Phi_{i}^{R}, \Phi_{i}^{I}, \varphi_{i}\right)$, one can express Eq. 
(5) in a compact form

$$
\begin{aligned}
& Z=\int D\left[\psi_{i}, \vec{\Omega}_{i}\right] e^{-\int_{0}^{\beta} d \tau L}, \\
& L=\sum_{i} \psi_{i}^{\dagger}\left(\partial_{\tau} \mathbf{I}-\mu \tau_{3}\right) \psi_{i}-t \sum_{\langle i j\rangle}\left(\psi_{i}^{\dagger} \tau_{3} \psi_{j}+H . c .\right) \\
& -i \sum_{i} \psi_{i}^{\dagger}\left(\vec{\Omega}_{i} \cdot \vec{\tau}\right) \psi_{i}+\frac{1}{4 u} \sum_{i} \operatorname{tr}\left(\vec{\Omega}_{i} \cdot \vec{\tau}\right)^{2} .
\end{aligned}
$$

Integrating over the pseudospin field $\vec{\Omega}_{i}$, Eq. (6) recovers the Hubbard model Eq. (4). Note that the U(1) slaverotor representation allows only the density-interaction channel including the $\tau_{3}$ matrix.

As discussed in the introduction, we disintegrate bare electrons into collective excitations and renormalized electrons in the following way

$$
\psi_{i}=e^{-i \phi_{1 i} \tau_{1}-i \phi_{2 i} \tau_{2}-i \phi_{3 i} \tau_{3}} \eta_{i} \equiv U_{i}^{\dagger} \eta_{i},
$$

where the two component spinor $\eta_{i}=\left(\begin{array}{c}\eta_{i+} \\ \eta_{i-}^{\dagger}\end{array}\right)$ can be considered to express renormalized electrons, and the $\mathrm{SU}(2)$ matrix field $U_{i}=\exp \left[i \sum_{k=1}^{3} \phi_{k i} \tau_{k}\right]$ collective bosons. Here $\exp \left[i \phi_{1 i} \tau_{1}\right]\left(\exp \left[i \phi_{2 i} \tau_{2}\right]\right)$ can be interpreted as a creation operator of an electron pair since it mixes a particle with a hole. If one takes only the $\phi_{1 i}$ phase field in the $U_{i}$ matrix field, i.e., $U_{i}=e^{i \phi_{1 i} \tau_{1}}$, he finds $\eta_{i+}=\cos \phi_{i} c_{i \uparrow}+i \sin \phi_{i} c_{i \downarrow}^{\dagger}$ and $\eta_{i-}^{\dagger}=i \sin \phi_{i} c_{i \uparrow}+\cos \phi_{i} c_{i \downarrow}^{\dagger}$ from $\eta_{i}=e^{i \phi_{1 i} \tau_{1}} \psi_{i}$. Considering the $\phi_{2 i}$ phase field, one obtains $\eta_{i+}=\cos \phi_{i} c_{i \uparrow}+\sin \phi_{i} c_{i \downarrow}^{\dagger}$ and $\eta_{i-}^{\dagger}=-\sin \phi_{i} c_{i \uparrow}+$ $\cos \phi_{i} c_{i \downarrow}^{\dagger}$ in the same way. On the other hand, $\exp \left[i \phi_{3 i} \tau_{3}\right]$ is identified with a creation operator of an electron charge, corresponding to the rotor variable in the $\mathrm{U}(1)$ slave-rotor representation.

Inserting the $\mathrm{SU}(2)$ slave-rotor decomposition Eq. (7) into the effective Lagrangian Eq. (6), we obtain

$$
\begin{aligned}
& Z=\int D\left[\eta_{i}, U_{i}, \vec{\Omega}_{i}\right] e^{-\int_{0}^{\beta} d \tau L}, \\
& L=\sum_{i} \eta_{i}^{\dagger}\left(\partial_{\tau} \mathbf{I}+U_{i} \partial_{\tau} U_{i}^{\dagger}-\mu U_{i} \tau_{3} U_{i}^{\dagger}\right) \eta_{i} \\
& -t \sum_{\langle i j\rangle}\left(\eta_{i}^{\dagger} U_{i} \tau_{3} U_{j}^{\dagger} \eta_{j}+H . c .\right) \\
& -i \sum_{i} \eta_{i}^{\dagger} U_{i}\left(\vec{\Omega}_{i} \cdot \vec{\tau}\right) U_{i}^{\dagger} \eta_{i}+\frac{1}{4 u} \sum_{i} \operatorname{tr}\left(\vec{\Omega}_{i} \cdot \vec{\tau}\right)^{2} .
\end{aligned}
$$

Performing the unitary transformation $\vec{\Omega}_{i} \cdot \vec{\tau} \rightarrow U_{i}^{\dagger}\left(\vec{\Omega}_{i}\right.$. $\vec{\tau}) U_{i}$ that makes the integration-measure invariant, Eq.
(8) reads

$$
\begin{aligned}
& Z=\int D\left[\eta_{i}, U_{i}, \vec{\Omega}_{i}\right] e^{-\int_{0}^{\beta} d \tau L}, \\
& L=\sum_{i} \eta_{i}^{\dagger}\left(\partial_{\tau} \mathbf{I}+U_{i} \partial_{\tau} U_{i}^{\dagger}-\mu U_{i} \tau_{3} U_{i}^{\dagger}\right) \eta_{i} \\
& -t \sum_{\langle i j\rangle}\left(\eta_{i}^{\dagger} U_{i} \tau_{3} U_{j}^{\dagger} \eta_{j}+H . c .\right) \\
& -i \sum_{i} \eta_{i}^{\dagger}\left(\vec{\Omega}_{i} \cdot \vec{\tau}\right) \eta_{i}+\frac{1}{4 u} \sum_{i} \operatorname{tr}\left(\vec{\Omega}_{i} \cdot \vec{\tau}\right)^{2} .
\end{aligned}
$$

Shifting $\vec{\Omega}_{i} \cdot \vec{\tau}$ as $\vec{\Omega}_{i} \cdot \vec{\tau}-i U_{i} \partial_{\tau} U_{i}^{\dagger}+i \mu U_{i} \tau_{3} U_{i}^{\dagger}$ that also makes the integration-measure invariant, we find the $\mathrm{SU}(2)$ slave-rotor representation of the Hubbard model as an extended version of its U(1) slave-rotor representation

$$
\begin{aligned}
& Z=\int D\left[\eta_{i}, U_{i}, \vec{\Omega}_{i}\right] e^{-\int_{0}^{\beta} d \tau L} \\
& L=\sum_{i} \eta_{i}^{\dagger}\left(\partial_{\tau} \mathbf{I}-i \vec{\Omega}_{i} \cdot \vec{\tau}\right) \eta_{i}-t \sum_{\langle i j\rangle}\left(\eta_{i}^{\dagger} U_{i} \tau_{3} U_{j}^{\dagger} \eta_{j}+H . c .\right) \\
& +\frac{1}{4 u} \sum_{i} \operatorname{tr}\left(-i U_{i} \partial_{\tau} U_{i}^{\dagger}+\vec{\Omega}_{i} \cdot \vec{\tau}+i \mu U_{i} \tau_{3} U_{i}^{\dagger}\right)^{2} .
\end{aligned}
$$

\section{B. Relation between the path integral formulation and canonical quantization}

One cautious person may suspect the above derivation because any decomposition method should always impose its associated constraint, but the path integral derivation does not seem to include an appropriate constraint. However, this guess is not correct because the above derivation imposes its constraint indeed. $[6,9]$

We start from the easy-axis anisotropy with $\vec{\Omega}_{i}=$ $\left(0,0, \varphi_{i}\right)$ and $U_{i}=e^{i \phi_{3} \tau_{3}}$ in order to check whether Eq. (10) recovers the $\mathrm{U}(1)$ slave-rotor Lagrangian [Eq. (3) with $\Delta=0$ ] in this limit. Inserting this easy-axis representation into Eq. (10), and performing the HS transformation for the last time-fluctuation term, we obtain

$$
\begin{aligned}
& Z=\int D\left[\eta_{i}, \phi_{3 i}, \varphi_{i}, L_{i}\right] \exp \left[-\int_{0}^{\beta} d \tau\left\{\sum_{i} \eta_{i}^{\dagger} \partial_{\tau} \eta_{i}\right.\right. \\
& -t \sum_{\langle i j\rangle}\left(\eta_{i}^{\dagger} e^{i \phi_{3 i} \tau_{3}} \tau_{3} e^{-i \phi_{3 j} \tau_{3}} \eta_{j}+H . c .\right) \\
& \left.\left.+\sum_{i}\left(\frac{u}{2} L_{i}^{2}-i L_{i}\left(\partial_{\tau} \phi_{3 i}-i \mu\right)+i \varphi_{i}\left(L_{i}-\eta_{i}^{\dagger} \tau_{3} \eta_{i}\right)\right)\right\}\right]
\end{aligned}
$$

where $\eta_{i}$ and $e^{-i \phi_{3 i} \tau_{3}}$ carry spin and charge degrees of freedom, respectively. In this expression the electron Hilbert space $\mid \psi_{i}>$ is decomposed into the direct product of the boson and fermion Hilbert spaces $\left|L_{i}>\otimes\right| \eta_{i}>$ according to the decomposition $\psi_{i}=e^{-i \phi_{3 i} \tau_{3}} \eta_{i}$, where $L_{i}$ 
represents an electron density at site $i$. Since this decomposition enlarges the original electron Hilbert space, the $\mathrm{U}(1)$ slave-rotor constraint $L_{i}=\eta_{i}^{\dagger} \tau_{3} \eta_{i}$ is introduced to reduce the enlarged Hilbert space into the original electron one. Then, $e^{-i \phi_{3 i} \tau_{3}}$ is identified with an annihilation operator of an electron charge in the Nambuspinor representation owing to the constraint $L_{i}=\eta_{i}^{\dagger} \tau_{3} \eta_{i}$ and the canonical relation $\left[L_{i}, \phi_{3 j}\right]=-i \delta_{i j}$ imposed by $-i L_{i} \partial_{\tau} \phi_{3 i}$. Actually, Eq. (11) is the starting point in the canonical quantization of the $\mathrm{U}(1)$ slave-rotor representation for the Hubbard model, Eq. (3) with $\Delta=0$ as discussed in the introduction. $[4,6,9]$ In this respect the above expression connects the canonical approach with the path integral representation.

In the same way as the above, performing the HS transformation for the last time-fluctuation term in Eq. (10)

$$
\begin{aligned}
& \frac{1}{4 u} \sum_{i} \operatorname{tr}\left(-i U_{i} \partial_{\tau} U_{i}^{\dagger}+\vec{\Omega}_{i} \cdot \vec{\tau}+i \mu U_{i} \tau_{3} U_{i}^{\dagger}\right)^{2} \\
& \rightarrow \sum_{i} \operatorname{tr}\left\{u M_{i}^{2}+i M_{i}\left(-i U_{i} \partial_{\tau} U_{i}^{\dagger}+\vec{\Omega}_{i} \cdot \vec{\tau}+i \mu U_{i} \tau_{3} U_{i}^{\dagger}\right)\right\}
\end{aligned}
$$

we obtain the following expression for the $\mathrm{SU}(2)$ slaverotor representation

$$
\begin{aligned}
& L=\sum_{i} \eta_{i}^{\dagger} \partial_{\tau} \eta_{i}-t \sum_{\langle i j\rangle}\left(\eta_{i}^{\dagger} U_{i} \tau_{3} U_{j}^{\dagger} \eta_{j}+H . c .\right) \\
& +\sum_{i}\left[\operatorname{tr}\left\{u M_{i}^{2}+i M_{i}\left(-i U_{i} \partial_{\tau} U_{i}^{\dagger}+i \mu U_{i} \tau_{3} U_{i}^{\dagger}\right)\right\}\right. \\
& \left.+i \vec{\Omega}_{i} \cdot\left(\operatorname{tr}\left[M_{i} \vec{\tau}\right]-\eta_{i}^{\dagger} \vec{\tau} \eta_{i}\right)\right]
\end{aligned}
$$

where $M_{i}$ is an $\mathrm{SU}(2)$ pseudospin matrix. In this $\mathrm{SU}(2)$ case the electron Hilbert space is represented as $\left|\psi_{i}>=\right| M_{i}>\otimes \mid \eta_{i}>$ according to the decomposition $\psi_{i}=U_{i}^{\dagger} \eta_{i}$. The Lagrange multiplier field $\vec{\Omega}_{i}$ confirms the local constraint $\operatorname{tr}\left[M_{i} \vec{\tau}\right]=\eta_{i}^{\dagger} \vec{\tau} \eta_{i}$. Using $M_{i}=\vec{N}_{i} \cdot \vec{\tau}$ where $\vec{N}_{i}$ corresponds to the pseudospin-density-wave order parameter, one can show that this is nothing but the standard relation $\vec{N}_{i}=\frac{1}{2} \eta_{i}^{\dagger} \vec{\tau} \eta_{i}$ as a natural extension of the $\mathrm{U}(1)$ slave-rotor constraint. $U_{i}^{\dagger}$ is identified with an annihilation operator of an electron pseudospin in the same way as the $\mathrm{U}(1)$ case.

\section{SU(2) slave-rotor gauge theory of the Hubbard model}

Using the HS transformation for the correlated hopping term in Eq. (10)

$$
\begin{aligned}
& -t\left(\eta_{i \alpha}^{\dagger} U_{i \alpha \beta} \tau_{3 \beta \gamma} U_{j \gamma \delta}^{\dagger} \eta_{j \delta}+H . c .\right) \\
& \rightarrow t\left[F_{i j \alpha \delta} E_{i j \delta \alpha}^{\dagger}+E_{i j \alpha \delta} F_{i j \delta \alpha}^{\dagger}\right. \\
& \left.-\left(\eta_{i \alpha}^{\dagger} F_{i j \alpha \delta} \eta_{j \delta}+U_{i \alpha \beta} \tau_{3 \beta \gamma} U_{j \gamma \delta}^{\dagger} E_{i j \delta \alpha}^{\dagger}\right)-H . c .\right]
\end{aligned}
$$

we find an effective Lagrangian of the Hubbard model

$$
\begin{aligned}
& Z=\int D\left[\eta_{i}, U_{i}, \vec{\Omega}_{i}, E_{i j}, F_{i j}\right] e^{-\int_{0}^{\beta} d \tau L}, \\
& L=L_{0}+L_{\eta}+L_{U}, \\
& L_{0}=t \sum_{\langle i j\rangle} \operatorname{tr}\left(F_{i j} E_{i j}^{\dagger}+E_{i j} F_{i j}^{\dagger}\right), \\
& L_{\eta}=\sum_{i} \eta_{i}^{\dagger}\left(\partial_{\tau} \mathbf{I}-i \vec{\Omega}_{i} \cdot \vec{\tau}\right) \eta_{i}-t \sum_{\langle i j\rangle}\left(\eta_{i}^{\dagger} F_{i j} \eta_{j}+H . c .\right), \\
& L_{U}=\frac{1}{4 u} \sum_{i} \operatorname{tr}\left(-i U_{i} \partial_{\tau} U_{i}^{\dagger}+\vec{\Omega}_{i} \cdot \vec{\tau}+i \mu U_{i} \tau_{3} U_{i}^{\dagger}\right)^{2} \\
& -t \sum_{\langle i j\rangle} \operatorname{tr}\left(U_{j}^{\dagger} E_{i j}^{\dagger} U_{i} \tau_{3}+H . c .\right),
\end{aligned}
$$

where $E_{i j}$ and $F_{i j}$ are HS matrix fields associated with hopping of $\eta_{i}$ fermions and $U_{i}$ bosons, respectively.

We make an ansatz for the hopping matrix fields as

$$
E_{i j} \approx E \exp \left[i \vec{a}_{i j} \cdot \vec{\tau}\right] \tau_{3}, \quad F_{i j} \approx F \exp \left[i \vec{a}_{i j} \cdot \vec{\tau}\right] \tau_{3}(14)
$$

where $E$ and $F$ are longitudinal modes (amplitudes) of the hopping parameters, and $\vec{a}_{i j}$ their transverse modes (phase fluctuations), considered to be spatial components of $\mathrm{SU}(2)$ gauge fields. The reason why we introduce the $\tau_{3}$ matrix is that the fermion sector $L_{\eta}$ should recover the original electron Lagrangian [Eq. (6)] as the slave-rotor representation does.

Inserting Eq. (14) into Eq. (13), we reach the $\mathrm{SU}(2)$ slave-rotor gauge theory of the Hubbard model for the metal-insulator transition

$$
\begin{aligned}
& Z=\int D\left[\eta_{i}, U_{i}, \vec{\Omega}_{i}, \vec{a}_{i j}\right] e^{-\int_{0}^{\beta} d \tau L}, \\
& L=L_{\eta}+L_{U}+4 t \sum_{\langle i j\rangle} E F, \\
& L_{\eta}=\sum_{i} \eta_{i}^{\dagger}\left(\partial_{\tau} \mathbf{I}-i \vec{\Omega}_{i} \cdot \vec{\tau}\right) \eta_{i}-t F \sum_{\langle i j\rangle}\left(\eta_{i}^{\dagger} e^{i \vec{a}_{i j} \cdot \vec{\tau}} \tau_{3} \eta_{j}+H . c .\right), \\
& L_{U}=\frac{1}{4 u} \sum_{i} \operatorname{tr}\left(-i U_{i} \partial_{\tau} U_{i}^{\dagger}+\vec{\Omega}_{i} \cdot \vec{\tau}+i \mu U_{i} \tau_{3} U_{i}^{\dagger}\right)^{2} \\
& -t E \sum_{\langle i j\rangle} \operatorname{tr}\left(U_{j}^{\dagger} \tau_{3} e^{-i \vec{a}_{i j} \cdot \vec{\tau}} U_{i} \tau_{3}+H . c .\right),
\end{aligned}
$$

where the unknown parameters such as the amplitudes of the hopping parameters $E, F$ and the $\mathrm{SU}(2)$ pseudospin order parameter $\vec{\Omega}_{i}$ should be determined by the following self-consistent mean-field equations

$$
\begin{aligned}
& 4 E=\left\langle\eta_{i}^{\dagger} \tau_{3} \eta_{j}+H . c .\right\rangle, \quad 4 F=\left\langle\operatorname{tr}\left(U_{j}^{\dagger} \tau_{3} U_{i} \tau_{3}+H . c .\right)\right\rangle \\
& \vec{\Omega}_{i}=i u\left\langle\eta_{i}^{\dagger} \vec{\tau} \eta_{i}\right\rangle-\frac{1}{2}\left\langle\operatorname{tr}\left[\vec{\tau}\left(-i U_{i} \partial_{\tau} U_{i}^{\dagger}+i \mu U_{i} \tau_{3} U_{i}^{\dagger}\right)\right]\right\rangle(16)
\end{aligned}
$$

as will be discussed later.

Compared with the U(1) slave-rotor effective action Eq. (3), the SU(2) slave-rotor effective action Eq. (15) exhibits more fruitful physics. Dynamics of collective 
charge fluctuations can be extracted from interacting electrons in the strong coupling approach as the $\mathrm{U}(1)$ case, but the structure of their dynamics is much richer. The effective boson action is represented as the nonlinear $\sigma$ model-type (its $\mathrm{CP}^{1}$ representation) with an effective chemical potential (the time component of an $\mathrm{SU}(2)$ gauge field) in the saddle-point approximation ignoring $\mathrm{SU}(2)$ gauge fluctuations while that in the $\mathrm{U}(1)$ slaverotor representation is governed by the boson-Hubbard model-type. The presence of additional collective charge fluctuations opens the possibility of new phases, as will be discussed in this paper.

Considering the fermion sector of the $\mathrm{SU}(2)$ slave-rotor representation, one cautious person can find the possibility of superconductivity in the Hubbard model. See the kinetic energy term of the fermion sector in Eq. (15). Because the $\mathrm{SU}(2)$ gauge matrix $W_{i j} \equiv \exp \left[-i \vec{a}_{i j} \cdot \vec{\tau}\right]$ has nonzero off-diagonal components, fermion pairing as the seed for superconductivity is naturally allowed. Local repulsive interactions (the Hubbard- $U$ term) appear to be local density and pairing fluctuations of the $\mathrm{SU}(2)$ matrix field $U_{i}$, governed by the nonlinear $\sigma$ model-type. These charge fluctuations generate $\mathrm{SU}(2)$ gauge fluctuations and couple to them, one of which corresponds to phase fluctuations of fermion pairs, observed in the kinetic energy term of the fermion sector. Since the SU(2) gauge fluctuations are controlled via the collective charge fluctuations, fermion-pairing fluctuations can be managed by the collective boson-dynamics, varying the coupling constant $u / t$ and hole concentration $\delta$. In summary, the $\mathrm{SU}(2)$ slave-rotor representation not only reveals the possibility of superconductivity in the Hubbard model, but also controls phase fluctuations of fermion pairs.

\section{U(1) charge-rotor gauge theory of the Hubbard model}

Since the structure of the $\mathrm{SU}(2)$ slave-rotor action [Eq. (15)] is complex to analyze, it is necessary to consider its easy-axis limit $\vec{\Omega}_{i} \cdot \vec{\tau} \equiv \varphi_{i} \tau_{3}, U_{i} \equiv \exp \left[i \phi_{3 i} \tau_{3}\right]$, and $\vec{a}_{i j} \cdot \vec{\tau} \equiv$ $a_{3 i j} \tau_{3}$. Then, Eq. (15) is reduced to the effective U(1) gauge Lagrangian in the slave-rotor representation[4-6, $8,9]$

$$
\begin{aligned}
& L_{\eta}=\sum_{i} \eta_{i}^{\dagger}\left(\partial_{\tau} \mathbf{I}-i \varphi_{i} \tau_{3}\right) \eta_{i} \\
& -t F \sum_{\langle i j\rangle}\left(\eta_{i}^{\dagger} e^{i a_{3 i j} \tau_{3}} \tau_{3} \eta_{j}+H . c .\right), \\
& L_{\phi}=\frac{1}{2 u} \sum_{i}\left(\partial_{\tau} \phi_{3 i}-\varphi_{i}-i \mu\right)^{2} \\
& -2 t E \sum_{\langle i j\rangle} \cos \left(\phi_{3 j}-\phi_{3 i}-a_{3 i j}\right) .
\end{aligned}
$$

Based on this slave-rotor effective Lagrangian, Florens and Georges performed a saddle-point analysis at half filling, and found a coherent-incoherent transition of the $\phi_{3 i}$ fields, identifying this transition with the Mott-Hubbard transition from a spin liquid Mott insulator to a Fermi liquid metal.[4] For our later discussion we perform the mean-field analysis of the U(1) slave-rotor theory.

For the saddle-point analysis one can resort to the large $N$ generalization for the boson sector replacing $e^{i \phi_{3 i}}$ with $X_{i \sigma}$

$$
\begin{aligned}
& L_{X}=\frac{1}{2 u} \sum_{i \sigma}\left(\partial_{\tau} X_{i \sigma}^{\dagger}-\left[\mu-\varphi_{i}\right] X_{i \sigma}^{\dagger}\right)\left(\partial_{\tau} X_{i \sigma}+\left[\mu-\varphi_{i}\right] X_{i \sigma}\right) \\
& -t E \sum_{i j \sigma} X_{j \sigma}^{\dagger} X_{i \sigma}+i \sum_{i} \lambda_{i}\left(\sum_{\sigma}\left|X_{i \sigma}\right|^{2}-1\right)
\end{aligned}
$$

where $\lambda_{i}$ is a Lagrange multiplier field to impose the uni-modular constraint for the rotor field $X_{i \sigma}$, and $\varphi_{i}$ is substituted for $i \varphi_{i}$. Based on the mean-field ansatz of $\varphi_{i}=\varphi_{0}, i \lambda_{i}=\lambda$, and $a_{3 i j}=0$, we find the mean-field free energy

$$
\begin{aligned}
& F_{M F}=-\frac{1}{\beta} \sum_{k, \omega} \ln \left[(i \omega)^{2}-\left(\varphi_{0}-F \epsilon_{k}^{\eta}\right)^{2}\right] \\
& +\frac{1}{\beta} \sum_{k, \nu} \sum_{\sigma} \ln \left[-\frac{\left(i \nu+\mu-\varphi_{0}\right)^{2}}{2 u}+\lambda+E \epsilon_{k}^{X}\right] \\
& +\sum_{k}(D E F-\mu \delta-\lambda),
\end{aligned}
$$

where $\omega(\nu)$ is the Matsubara frequency for fermions (bosons) with inverse temperature $\beta=1 / T$, and $\epsilon_{k}^{\eta(X)}=$ $-2 t\left(\cos k_{x}+\cos k_{y}\right)$ is the fermion (boson) dispersion that originates from the electron dispersion. $\delta$ is hole concentration.

Minimizing the free energy with respect to $E, F, \varphi_{0}, \lambda$, and $\mu$, we find the self-consistent saddle-point equations for the mean-field parameters

$$
\begin{aligned}
& D E=\int_{-D}^{D} d \epsilon D(\epsilon) \frac{1}{\beta} \sum_{\omega} \frac{2\left(\varphi_{0}-F \epsilon\right) \epsilon}{(i \omega)^{2}-\left(\varphi_{0}-F \epsilon\right)^{2}}, \\
& D F=-\int_{-D}^{D} d \epsilon D(\epsilon) \frac{1}{\beta} \sum_{\nu \sigma} \frac{\epsilon}{-\frac{\left(i \nu+\mu-\varphi_{0}\right)^{2}}{2 u}+\lambda+E \epsilon}, \\
& \int_{-D}^{D} d \epsilon D(\epsilon) \frac{1}{\beta} \sum_{\omega} \frac{2\left(\varphi_{0}-F \epsilon\right)}{(i \omega)^{2}-\left(\varphi_{0}-F \epsilon\right)^{2}} \\
& =-\int_{-D}^{D} d \epsilon D(\epsilon) \frac{1}{\beta} \sum_{\nu \sigma} \frac{\frac{i \nu+\mu-\varphi_{0}}{u}}{-\frac{\left(i \nu+\mu-\varphi_{0}\right)^{2}}{2 u}+\lambda+E \epsilon} \\
& 1=\int_{-D}^{D} d \epsilon D(\epsilon) \frac{1}{\beta} \sum_{\nu \sigma} \frac{1}{-\frac{\left(i \nu+\mu-\varphi_{0}\right)^{2}}{2 u}+\lambda+E \epsilon}, \\
& -\delta=\int_{-D}^{D} d \epsilon D(\epsilon) \frac{1}{\beta} \sum_{\nu \sigma} \frac{\frac{i \nu+\mu-\varphi_{0}}{u}}{-\frac{\left(i \nu+\mu-\varphi_{0}\right)^{2}}{2 u}+\lambda+E \epsilon} .
\end{aligned}
$$

Here $\sum_{k}$ is replaced with $\int_{-D}^{D} d \epsilon D(\epsilon)$, where $D$ is the half bandwidth and $D(\epsilon)$ is the density of states for an electron band.

Performing the Matsubara frequency summation and energy integration with the constant density of states 
$D(\epsilon)=1 /(2 D)$ in Eq. (20), we obtain the algebraic equations for the mean-field parameters $E, F, \varphi_{0}, \lambda$, and $\mu$ at zero temperature

$$
\begin{aligned}
& E=\frac{1}{2}\left[1-\left(\frac{\varphi_{0}}{D F}\right)^{2}\right], \\
& F=\frac{1}{3(D E)^{2}}[(2 \lambda-D E) \sqrt{2 u(\lambda+D E)} \\
& \left.-\left(2 \lambda-E \epsilon_{-}\right) \sqrt{2 u\left(\lambda+E \epsilon_{-}\right)}\right] \\
& -\frac{\varphi_{0}}{D F}=1-\frac{\lambda}{D E}+\frac{1}{D E} \frac{\left(\mu-\varphi_{0}\right)^{2}}{2 u}, \\
& 1=\frac{\sqrt{2 u(\lambda+D E)}-\sqrt{2 u\left(\lambda+E \epsilon_{-}\right)}}{D E}, \\
& \delta=1-\frac{\lambda}{D E}+\frac{1}{D E} \frac{\left(\mu-\varphi_{0}\right)^{2}}{2 u},
\end{aligned}
$$

where $\epsilon_{-}$is given by $\epsilon_{-}=\frac{1}{E}\left[\frac{\left(\mu-\varphi_{0}\right)^{2}}{2 u}-\lambda\right]$. From the third and fifth equations we find $\varphi_{0}=-D F \delta$ and $\mu=-D F \delta-\sqrt{2 u[\lambda-D E(1-\delta)]}$. Inserting these expressions into the above equations, we obtain

$$
\begin{aligned}
& E=\frac{1-\delta^{2}}{2}, \\
& F=\frac{1}{3(D E)^{2}}[(2 \lambda-D E) \sqrt{2 u(\lambda+D E)} \\
& -[2 \lambda+D E(1-\delta)] \sqrt{2 u[\lambda-D E(1-\delta)]}, \\
& 1=\frac{\sqrt{2 u(\lambda+D E)}-\sqrt{2 u[\lambda-D E(1-\delta)]}}{D E} .
\end{aligned}
$$

Condensation of the $\phi_{3 i}$ bosons occurs when their excitation gap closes, given by $\mu_{c}-\varphi_{0 c}=0$ or $\lambda_{c}-D E_{c}(1-\delta)=$ 0 that determines the Mott-Hubbard critical point

$$
\frac{u_{c}}{D}=\frac{1}{4}\left(\frac{1-\delta^{2}}{2-\delta}\right)
$$

with $F_{c}=(1-2 \delta) / 3$ in the mean-field approximation. This means that the spin liquid Mott insulator $\left(\left\langle e^{i \phi_{3 i}}\right\rangle=0\right)$ appears in $u / D>u_{c} / D$, and the Fermi liquid metal $\left(\left\langle e^{i \phi_{3 i}}\right\rangle \neq 0\right)$ arises in $u / D<u_{c} / D$. It is important to notice that since the slave-rotor decomposition is meaningful in the case of $E>0$ and $F>0$, the critical value of $F_{c}=(1-2 \delta) / 3$ indicates that this representation is valid when hole concentration is relatively small, here $\delta<1 / 2$.

\section{E. Spin liquid Mott insulator away from half filling}

One would be surprised at this result that the slaverotor theory allows the spin liquid Mott insulator away from half filling, in contrast with the common belief that hole doping to a Mott insulator is expected to cause a metal. Hole doping to a Mott insulator shifts the chemical potential from the middle of the charge gap generated by the energy cost $(u)$ for double occupancy to the top of the lower Hubbard band.[10] Since the density of states at the top of the lower Hubbard band is nonzero, the resulting state is expected to be a metallic phase. Actually, the $t-J$ model studies find a metallic phase at all fillings, even arbitrarily close to half filling.[11]

Recently, Choy and Phillips claimed that this common belief may not be right in the Hubbard model. Doped Mott insulators can be insulators.[10] They demonstrated that hole localization can obtain because the chemical potential lies in a pseudogap which has vanishing density of states at zero temperature. The pseudogap in the doped Mott insulator results from short-range antiferromagnetic correlations corresponding to the nearest-neighbor singlet-triplet splitting. They showed that the pseudogap vanishes without the triplet contribution which lies above the chemical potential, claiming that the presence of the upper Hubbard band is crucial for the pseudogap. More fundamentally, they proposed that physics is sensitive to the order of limits of $u \rightarrow \infty$ and $L \rightarrow \infty$, where $L$ is the system size. They suggested the relevance length scale $\xi_{d o}$ for the pseudogap, where $\xi_{d o}$ represents the average distance between doubly occupied sites. They claimed that the order of $u \rightarrow \infty$ and $L \rightarrow \infty$ results in $\xi_{d o}>L$, metallic transport while $\xi_{d o}<L$ and localization obtains in the reverse order of limits, provided that $n_{h} \xi_{d o}^{2}<L$, $n_{h}=x(L / a)^{2}$ the number of holes with lattice spacing $a$. Furthermore, considering the scaling form $Z \sim L^{-(t / u)^{p}}$ with $p>0$ for the one-hole quasiparticle weight $Z$, they addressed that the discrepancy between the $t-J$ and Hubbard results implies the non-commutativity of the order of limits. In the $t-J$ model (no double occupancy) corresponding to $u \rightarrow \infty$ and $L \rightarrow \infty, Z$ remains finite. But, $Z$ vanishes in the reverse order of limits (Hubbard model).

The slave-rotor theory admits double occupancy. In addition, it was demonstrated that the exchange energy scale $J \sim t^{2} / u$ is indeed generated at the level of oneparticle properties, where it cuts the divergence of the effective mass at the Mott transition driven by $u$. [4] Actually, the single-particle spectral weight is enhanced near the chemical potential with vanishing density of states at the chemical potential, decreasing dimensionality from infinite dimensions near the Mott critical point at half filling, which is associated with the exchange energy scale.[4]

To see the mechanism for the spin liquid Mott insulator away from half filling in the slave-rotor theory more concretely, we consider the effective chemical potential in the boson sector of Eq. (18) carefully. It is important to notice that the effective chemical potential for the rotor condensation is given by $\mu-\varphi_{0}$ instead of $\varphi_{0}$ or $\mu$. The point is that the effective chemical potential $\mu-\varphi_{0}=-\sqrt{2 u[\lambda-D E(1-\delta)]}$ away from half filling shows essentially the same behavior as the chemical potential $\mu=-\sqrt{2 u[\lambda-D E]}$ with $\varphi_{0}=0$ at half filling although $\mu=-D F \delta-\sqrt{2 u[\lambda-D E(1-\delta)]}$ and $\varphi_{0}=-D F \delta$ away from half filling have doping dependencies proportional to hole concentration.[12] Considering physics of the slave-rotor variable, this behavior of 
the effective chemical potential can be understood. The $\mathrm{U}(1)$ slave-rotor variable raises or lowers the local density of fermions. Thus, hole concentration has nothing to do with the density of slave-rotor bosons. This is in contrast with the slave-boson representation of the $t-J$ model, where hole concentration is exactly the same as the density of slave-bosons in the saddle-point approximation. In the $t-J$ model the single occupancy constraint of $f_{i \sigma}^{\dagger} f_{i \sigma}+b_{i}^{\dagger} b_{i}=1$ with spinon $f_{i \sigma}$ and holon $b_{i}$ results in $\left\langle f_{i \sigma}^{\dagger} f_{i \sigma}\right\rangle=1-\delta$ and $\left\langle b_{i}^{\dagger} b_{i}\right\rangle=\delta$, thus causing the condensation of holons due to the holon chemical potential as soon as holes are doped. On the other hand, there is no such constraint in the slave-rotor representation, thus the density of slave-rotor bosons does not follow hole concentration. Hole doping changes the density of fermions only $\left(\left\langle\eta_{i}^{\dagger} \tau_{3} \eta_{i}\right\rangle=-\delta\right)$, reflected in the doping dependence of the effective chemical potential $\varphi_{0}=-D F \delta$ for fermions. The effect of hole doping on the boson dynamics is just to modify the bandwidth from $D E$ to $D E(1-\delta)$. As a result, the slave-rotor excitations can be gapped away from half filling.

Compared with the work of Choy and Phillips, [10] the slave-rotor theory seems to have some similarities. The effective "charge" chemical potential $\mu-\varphi_{0}$, associated with softening of local charge fluctuations, can lie in the gap away from half filling in the large $u / t$ limit, similar to the half-filled case, while the effective "spin" chemical potential $\varphi_{0}$ shows the conventional behavior as the Fermi liquid or the fermion dynamics in the $\mathrm{U}(1)$ slave-boson theory of the $t-J$ model. Considering that the slaverotor description can capture local antiferromagnetic correlations of $J$, allowing double occupancy seems to play a crucial role for the emergence of the Mott insulator away from half filling.

\section{F. U(1) pair-rotor gauge theory of the Hubbard model}

Now we consider the easy-plane limit $\vec{\Omega}_{i} \cdot \vec{\tau} \equiv \Phi_{i}^{R} \tau_{1}$, $U_{i} \equiv \exp \left[i \phi_{1 i} \tau_{1}\right]$, and $\vec{a}_{i j} \cdot \vec{\tau} \equiv a_{1 i j} \tau_{1}$. Then, we find another effective $\mathrm{U}(1)$ gauge Lagrangian[13]

$$
\begin{aligned}
& L_{\eta}=\sum_{i} \eta_{i}^{\dagger}\left(\partial_{\tau} \mathbf{I}-i \Phi_{i}^{R} \tau_{1}\right) \eta_{i} \\
& -t F \sum_{\langle i j\rangle}\left(\eta_{i}^{\dagger} e^{i a_{1 i j} \tau_{1}} \tau_{3} \eta_{j}+H . c .\right), \\
& L_{\phi}=\frac{1}{2 u} \sum_{i}\left(\partial_{\tau} \phi_{1 i}-\Phi_{i}^{R}\right)^{2} \\
& -2 t E \sum_{\langle i j\rangle} \cos \left(\phi_{1 j}+\phi_{1 i}-a_{1 i j}\right) .
\end{aligned}
$$

We note that Eq. (24) can be reduced to Eq. (6) with $\vec{\Omega}_{i} \cdot \vec{\tau} \equiv \Phi_{i}^{R} \tau_{1}$ after the gauge transformation of $\Phi_{i}^{R} \rightarrow \Phi_{i}^{R}+\partial_{\tau} \phi_{1 i}, a_{1 i j} \rightarrow a_{1 i j}+\phi_{1 i}+\phi_{1 j}$, and Eq. (7) without $\phi_{2 i}$ and $\phi_{3 i}$ are utilized. If the $\tau_{3}$ matrix is not used in Eq. (14), the hopping term in $L_{\phi}$ vanishes, and Eq. (6) cannot be recovered from Eq. (24). Ignoring gauge fluctuations, and replacing $\Phi_{i}^{R}$ with $\Phi_{0}$ as the mean-field approximation, we obtain the on-site pairing order parameter given by $\Phi_{0}=i u\left\langle\eta_{i}^{\dagger} \tau_{1} \eta_{i}\right\rangle+\left\langle\partial_{\tau} \phi_{1 i}\right\rangle$. It turns out to be zero because double occupancy costs too much energy. Thus, there is no phase transition in the fermion Lagrangian at half filling as the case of the easyaxis limit.

To examine the boson Lagrangian in the mean-field level, we resort to the large $N$ generalization as the $\mathrm{U}(1)$ slave-rotor representation. Introducing the $N$ component rotor field $Y_{i \sigma}$, we rewrite $L_{\phi}$ in Eq. (24) as

$$
\begin{aligned}
& L_{Y}=\frac{1}{2 u} \sum_{i \sigma}\left(\partial_{\tau} Y_{i \sigma}^{\dagger}\right)\left(\partial_{\tau} Y_{i \sigma}\right)-t E \sum_{\langle i j\rangle \sigma}\left(Y_{i \sigma}^{\dagger} Y_{j \sigma}^{\dagger}+Y_{j \sigma} Y_{i \sigma}\right) \\
& +i \sum_{i} \lambda_{i}\left(\sum_{\sigma}\left|Y_{i \sigma}\right|^{2}-1\right),
\end{aligned}
$$

where $\lambda_{i}$ is a Lagrange multiplier field for the unimodular constraint. If we represent Eq. (25) in terms of $Y_{i \sigma}=R_{i \sigma}+i I_{i \sigma}\left(Y_{i \sigma}^{\dagger}=R_{i \sigma}-i I_{i \sigma}\right)$, we obtain the following expression for the mean-field Lagrangian of collective pair excitations

$$
\begin{aligned}
& L_{Y}=\frac{1}{2 u} \sum_{i \sigma}\left[\left(\partial_{\tau} R_{i \sigma}\right)^{2}+\left(\partial_{\tau} I_{i \sigma}\right)^{2}\right] \\
& -2 t E \sum_{\langle i j\rangle \sigma}\left(R_{i \sigma} R_{j \sigma}+I_{i \sigma} I_{j \sigma}\right)+i \sum_{i} \lambda_{i}\left[\sum_{\sigma}\left(R_{i \sigma}^{2}+I_{i \sigma}^{2}\right)-1\right] .
\end{aligned}
$$

One can find that Eq. (26) is exactly the same as Eq. (18) if we rewrite Eq. (18) in terms of $X_{i \sigma}=R_{i \sigma}+i I_{i \sigma}$. Thus, the mean-field analysis in the previous slave-rotor theory can be directly applied to Eq. (25). This leads us to conclude that both the $\phi_{3 i}$ and $\phi_{1 i}$ fields are simultaneously incoherent in $(u / t)>(u / t)_{0}$, and coherent in $(u / t)<(u / t)_{0}$ at half filling, where $(u / t)_{0}$ is the critical value for the Mott transition obtained in Eq. (23). This originates from $\varphi_{0}=0$ at half filling and $\mu_{c}=0$ at the Mott-Hubbard critical point, making Eq. (18) exactly the same as $L_{\phi}$ in Eq. (24). Fundamentally, the reason why both fields should be coherent simultaneously is the presence of the $\mathrm{SU}(2)$ pseudospin symmetry at half filling. The slave-rotor action should be symmetric (invariant) under the transformation $\phi_{1 i} \longleftrightarrow \phi_{3 i}$ at half filling in the mean-field approximation.

\section{MEAN-FIELD ANALYSIS OF THE SU(2) SLAVE-ROTOR THEORY}

So far, we discussed that only two phases are expected to appear at half filling in the context of the $\mathrm{SU}(2)$ slaverotor theory, corresponding to the spin liquid Mott insulator with $\left\langle e^{i \phi_{3 i}}\right\rangle=0,\left\langle e^{i \phi_{1 i}}\right\rangle=0$ and the Fermi liquid 
metal with $\left\langle e^{i \phi_{3 i}}\right\rangle \neq 0,\left\langle e^{i \phi_{1 i}}\right\rangle \neq 0$, respectively. There is no intermediate phase because the presence of the $\mathrm{SU}(2)$ pseudospin symmetry makes both phase fields simultaneously coherent or incoherent. Away from half filling an intermediate phase, characterized by condensation of only one kind of bosons, is expected to arise because the $\mathrm{SU}(2)$ pseudospin symmetry is broken explicitly due to hole doping, reflected in the chemical potential term.

For the saddle-point analysis we consider the following mean-field Lagrangian from Eq. (15), where $\mathrm{SU}(2)$ gauge fluctuations are ignored $\left(e^{i \vec{a}_{i j} \cdot \vec{\tau}} \rightarrow I\right)$, and the easy-axis anisotropy for the $\mathrm{SU}(2)$ pseudospin order parameter is naturally chosen $\left(\vec{\Omega}_{i} \cdot \vec{\tau} \rightarrow-i \varphi_{0} \tau_{3}\right)$,

$$
\begin{aligned}
& L=L_{\eta}+L_{U}+4 t \sum_{\langle i j\rangle} E F, \\
& L_{\eta}=\sum_{i} \eta_{i}^{\dagger}\left(\partial_{\tau} \mathbf{I}-\varphi_{0} \tau_{3}\right) \eta_{i}-t F \sum_{\langle i j\rangle}\left(\eta_{i}^{\dagger} \tau_{3} \eta_{j}+H . c .\right) \\
& L_{U}=\frac{1}{4 u} \sum_{i} \operatorname{tr}\left(-i U_{i} \partial_{\tau} U_{i}^{\dagger}-i \varphi_{0} \tau_{3}+i \mu U_{i} \tau_{3} U_{i}^{\dagger}\right)^{2} \\
& -t E \sum_{\langle i j\rangle} \operatorname{tr}\left(U_{j}^{\dagger} \tau_{3} U_{i} \tau_{3}+H . c .\right) .
\end{aligned}
$$

The first assumption to ignore the $\mathrm{SU}(2)$ gauge fluctuations is the simplest mean-field ansatz. The second assumption of the easy-axis anisotropy for the $\mathrm{SU}(2)$ pseudospin order parameter can be justified from the self-consistent mean-field condition $\vec{\Omega}_{i}=i u\left\langle\eta_{i}^{\dagger} \vec{\tau} \eta_{i}\right\rangle-$ $\frac{1}{2}\left\langle\operatorname{tr}\left[\vec{\tau}\left(-i U_{i} \partial_{\tau} U_{i}^{\dagger}+i \mu U_{i} \tau_{3} U_{i}^{\dagger}\right)\right]\right\rangle$. The first term in this expression becomes zero for $\tau_{1}$ and $\tau_{2}$ because double occupancy costs too much energy. The second term also vanishes for $\tau_{1}$ and $\tau_{2}$ because this term contains pseudospin-flipping terms in the representation $U_{i}=$ $\left(\begin{array}{cc}z_{i \uparrow} & -z_{i \downarrow}^{\dagger} \\ z_{i \downarrow} & z_{i \uparrow}^{\dagger}\end{array}\right)$ of the $\mathrm{SU}(2)$ slave-rotor matrix, but the boson Lagrangian $L_{U}$ has no pseudospin-flipping terms as will be seen below. Particulary, the second mean-field ansatz has important physical implication for the $\mathrm{SU}(2)$ slave-rotor theory that the $\mathrm{SU}(2)$ pseudospin symmetry is broken away from half filling.

Representing Eq. (27) in terms of the $z_{i \sigma}$ field, we obtain the following expression

$$
\begin{aligned}
& L=L_{\eta}+L_{U}+2 t \sum_{\langle i j\rangle} E F \\
& L_{\eta}=\sum_{i} \eta_{i}^{\dagger}\left(\partial_{\tau} \mathbf{I}-\varphi_{0} \tau_{3}\right) \eta_{i}-t F \sum_{\langle i j\rangle}\left(\eta_{i}^{\dagger} \tau_{3} \eta_{j}+H . c .\right) \\
& L_{z}=\frac{1}{2 u} \sum_{i \sigma}\left(\partial_{\tau} z_{i \sigma}^{\dagger}-\left[\mu-\sigma \varphi_{0}\right] z_{i \sigma}^{\dagger}\right)\left(\partial_{\tau} z_{i \sigma}+\left[\mu-\sigma \varphi_{0}\right] z_{i \sigma}\right) \\
& -t E \sum_{\langle i j\rangle \sigma}\left(\sigma z_{i \sigma}^{\dagger} z_{j \sigma}+H . c .\right)+\lambda \sum_{i}\left(\sum_{\sigma}\left|z_{i \sigma}\right|^{2}-1\right)
\end{aligned}
$$

where $2 E$ and $\tau_{3}$ are replaced with $E$ and $\sigma= \pm$, respectively. Although the $\mathrm{SU}(2)$ slave-rotor mean-field
Lagrangian Eq. (28) seems to be similar to the U(1) charge-rotor mean-field Lagrangian Eq. (17) with Eq. (18), there is a crucial difference between them. The most important ingredient in Eq. (28) is the pseudospindependent chemical potential $\sigma \varphi_{0}$ in the boson sector. We show that this results in the condensation of only one kind of bosons, allowing an intermediate phase away from half filling. Since the renormalized dispersion of the $z_{i \sigma}$ bosons depends on the pseudospin component, the lowest energy of the $z_{i \uparrow}$ bosons lies at momentum $(0,0)$ while that of the $z_{i \downarrow}$ bosons appears at momentum $(\pi, \pi)$. There are no pseudospin-flipping terms in the boson Lagrangian, thus the pseudospin-flipping terms such as $\left\langle z_{i \uparrow}^{\dagger} \partial_{\tau} z_{i \downarrow}\right\rangle$ and $\left\langle z_{i \uparrow}^{\dagger} z_{i \downarrow}\right\rangle$ appearing in the self-consistent condition for the SU(2) pseudospin order parameter $\vec{\Omega}_{i}$ should be zero, justifying our assumption of the easy-axis anisotropy for the order parameter.

Integrating out the $\eta_{i}$ and $z_{i \sigma}$ fields, we obtain the mean-field free energy

$$
\begin{aligned}
& F_{M F}=-\frac{1}{\beta} \sum_{k, \omega} \ln \left[(i \omega)^{2}-\left(\varphi_{0}-F \epsilon_{k}^{\eta}\right)^{2}\right] \\
& +\frac{1}{\beta} \sum_{k, \nu} \sum_{\sigma} \ln \left[-\frac{\left(i \nu+\mu-\sigma \varphi_{0}\right)^{2}}{2 u}+\lambda+E \sigma \epsilon_{k}^{z}\right] \\
& +\sum_{k}(D E F-\mu \delta-\lambda) .
\end{aligned}
$$

Since the $z_{i \downarrow}$ bosons have their energy minima at momentum $Q=(\pi, \pi)$, we shift the momentum $k$ of the $z_{i \downarrow}$ boson to $k+Q$. Then, the mean-field free energy is given by

$$
\begin{aligned}
& F_{M F}=-\frac{1}{\beta} \sum_{k, \omega} \ln \left[(i \omega)^{2}-\left(\varphi_{0}-F \epsilon_{k}^{\eta}\right)^{2}\right] \\
& +\frac{1}{\beta} \sum_{k, \nu} \sum_{\sigma} \ln \left[-\frac{\left(i \nu+\mu-\sigma \varphi_{0}\right)^{2}}{2 u}+\lambda+E \epsilon_{k}^{z}\right] \\
& +\sum_{k}(D E F-\mu \delta-\lambda)
\end{aligned}
$$

where the $\sigma$ symbol disappears in the boson dispersion. Compared with the U(1) charge-rotor free energy Eq. (19), Eq. (30) allows the pseudospin-dependent chemical potential $\sigma \varphi_{0}$ in the boson free energy. The charge chemical potentials appear to be $\mu-\varphi_{0}$ for the $z_{i \uparrow}$ field and $\mu+\varphi_{0}$ for the $z_{i \downarrow}$ field with the spin chemical potential $\varphi_{0}$ in the $\mathrm{SU}(2)$ slave-rotor theory while the charge chemical potential is given by $\mu-\varphi_{0}$ for the $e^{i \phi_{3 i}}$ field with the spin chemical potential $\varphi_{0}$ in the U(1) charge-rotor theory.

Minimizing the free energy with respect to $E, F, \varphi_{0}, \lambda$, and $\mu$, we find the self-consistent saddle-point equations 
for all mean-field parameters

$$
\begin{aligned}
& D E=\int_{-D}^{D} d \epsilon D(\epsilon) \frac{1}{\beta} \sum_{\omega} \frac{2\left(\varphi_{0}-F \epsilon\right) \epsilon}{(i \omega)^{2}-\left(\varphi_{0}-F \epsilon\right)^{2}}, \\
& D F=-\int_{-D}^{D} d \epsilon D(\epsilon) \frac{1}{\beta} \sum_{\nu} \sum_{\sigma} \frac{\epsilon}{-\frac{\left(i \nu+\mu-\sigma \varphi_{0}\right)^{2}}{2 u}+\lambda+E \epsilon}, \\
& \int_{-D}^{D} d \epsilon D(\epsilon) \frac{1}{\beta} \sum_{\omega} \frac{2\left(\varphi_{0}-F \epsilon\right)}{(i \omega)^{2}-\left(\varphi_{0}-F \epsilon\right)^{2}} \\
& =-\int_{-D}^{D} d \epsilon D(\epsilon) \frac{1}{\beta} \sum_{\nu} \sum_{\sigma} \frac{\sigma \frac{i \nu+\mu-\sigma \varphi_{0}}{u}}{-\frac{\left(i \nu+\mu-\sigma \varphi_{0}\right)^{2}}{2 u}+\lambda+E \epsilon}, \\
& 1=\int_{-D}^{D} d \epsilon D(\epsilon) \frac{1}{\beta} \sum_{\nu} \sum_{\sigma} \frac{1}{-\frac{\left(i \nu+\mu-\sigma \varphi_{0}\right)^{2}}{2 u}+\lambda+E \epsilon}, \\
& -\delta=\int_{-D}^{D} d \epsilon D(\epsilon) \frac{1}{\beta} \sum_{\nu} \sum_{\sigma} \frac{\frac{i \nu+\mu-\sigma \varphi_{0}}{u}}{-\frac{\left(i \nu+\mu-\sigma \varphi_{0}\right)^{2}}{2 u}+\lambda+E \epsilon}(31)
\end{aligned}
$$

One can see from the denominator of the boson propagator that condensation of the $z_{i \sigma}$ bosons occurs when their excitation gap closes, given by $-\mu_{c}+\varphi_{0 c}=0$ for the $z_{i \uparrow}$ bosons at momentum $(0,0)$ and $-\mu_{c}-\varphi_{0 c}=0$ for the $z_{i \downarrow}$ bosons at momentum $(\pi, \pi)$, respectively. For both bosons to be condensed simultaneously, $\mu_{c}=0$ and $\varphi_{0 c}=0$ should be satisfied. Actually, this happens at half filling where both bosons have exactly the same transition point given by $\lambda_{c}=E_{c} D$ as a result of the $\mathrm{SU}(2)$ pseudospin symmetry. Away from half filling the effective chemical potential $\varphi_{0}$ becomes nonzero, causing the $\mathrm{SU}(2)$ pseudospin symmetry breaking not spontaneously but explicitly because it is pseudospin-dependent. As a result, the $z_{i \uparrow}$ bosons should be condensed before the condensation of the $z_{i \downarrow}$ bosons occurs. Only one kind of bosons can be condensed in the intermediate range of $u / t$, corresponding to the intermediate phase mentioned before.

Performing the Matsubara frequency summation and energy integration in Eq. (31), we find the following expressions for the mean-field parameters $E, F, \varphi_{0}, \lambda$, and $\mu$ at zero temperature

$$
\begin{aligned}
& E=\frac{1}{2}\left[1-\left(\frac{\varphi_{0}}{D F}\right)^{2}\right] \\
& F=\frac{1}{6(D E)^{2}}[(2 \lambda-D E) \sqrt{2 u(\lambda+D E)} \\
& \left.-\left(2 \lambda-E \epsilon_{-}\right) \sqrt{2 u\left(\lambda+E \epsilon_{-}\right)}\right] \\
& +\frac{1}{6(D E)^{2}}[(2 \lambda-D E) \sqrt{2 u(\lambda+D E)} \\
& \left.-\left(2 \lambda-E \epsilon_{+}\right) \sqrt{2 u\left(\lambda+E \epsilon_{+}\right)}\right] \\
& \varphi_{0}\left(\mu+\frac{E}{F} u\right)=0, \\
& 1=\frac{\sqrt{2 u(\lambda+D E)}-\sqrt{2 u\left(\lambda+E \epsilon_{-}\right)}}{2 D E} \\
& +\frac{\sqrt{2 u(\lambda+D E)}-\sqrt{2 u\left(\lambda+E \epsilon_{+}\right)}}{2 D E}, \\
& \delta=1-\frac{\lambda}{D E}+\frac{1}{2 D E}\left[\frac{\left(\mu-\varphi_{0}\right)^{2}}{2 u}+\frac{\left(\mu+\varphi_{0}\right)^{2}}{2 u}\right]
\end{aligned}
$$

where $\epsilon_{ \pm}$is given by $\epsilon_{ \pm}=\frac{1}{E}\left[\frac{\left(\mu \pm \varphi_{0}\right)^{2}}{2 u}-\lambda\right]$. One can see the validity of these equations performing the limit of $\delta \rightarrow 0$. We obtain $\varphi_{0}=0$ at half filling from the third equation. This causes $\mu=-\sqrt{2 u(\lambda-D E)}$ in the fifth equation. Using the expressions for $\epsilon_{ \pm}$with $\mu=$ $-\sqrt{2 u(\lambda-D E)}$ and $\varphi_{0}=0$, one can find $\epsilon_{ \pm}=-D$, reproducing the mean-field equations for the half-filled case.[12]

As soon as holes are doped, the chemical potential $\mu$ jumps from $\mu=-\sqrt{2 u(\lambda-D E)}$ to $\mu=-(E / F) u$. Decreasing the Hubbard interactions with this chemical potential, one can find the Mott-Hubbard transition point, where the $z_{i \uparrow}$ bosons begin to be condensed. The $z_{i \uparrow}$ condensation occurs when $\lambda_{c}+E_{c} \epsilon_{-}=0$ is satisfied. From the analytic expressions in Eq. (32) with $\epsilon_{ \pm}$, we obtain the following conditions

$$
\begin{aligned}
& E_{c}=\frac{1}{2}\left[1-\frac{E_{c}^{2}}{F_{c}^{4}}\left(\frac{u_{c}}{D}\right)^{2}\right], \\
& 3 F_{c} E_{c}^{2}=\left(2 \frac{\lambda_{c}}{D}-E_{c}\right) \sqrt{2 \frac{u_{c}}{D}\left(\frac{\lambda_{c}}{D}+E_{c}\right)} \\
& -\frac{u_{c}}{D} \frac{E_{c}}{F_{c}}\left[3 \frac{\lambda_{c}}{D}-2 \frac{u_{c}}{D}\left(\frac{E_{c}}{F_{c}}\right)^{2}\right], \\
& \left(E_{c}+\frac{u_{c}}{D} \frac{E_{c}}{F_{c}}\right)^{2}=2 \frac{u_{c}}{D}\left(\frac{\lambda_{c}}{D}+E_{c}\right), \\
& \frac{\lambda_{c}}{D}=(1-\delta) E_{c}+\frac{u_{c}}{D}\left(\frac{E_{c}}{F_{c}}\right)^{2},
\end{aligned}
$$

determining the critical value $u_{c} / D$ for the MottHubbard transition in the SU(2) slave-rotor theory away from half filling. We note that the above equations for the Mott critical point do not recover the half-filled case owing to the chemical potential jump. From these equations we find $u_{c} / D \approx 0.170$ at $\delta=0.010, u_{c} / D \approx 0.171$ at $\delta=0.050$, and $u_{c} / D \approx 0.173$ at $\delta=0.100$. The value of 


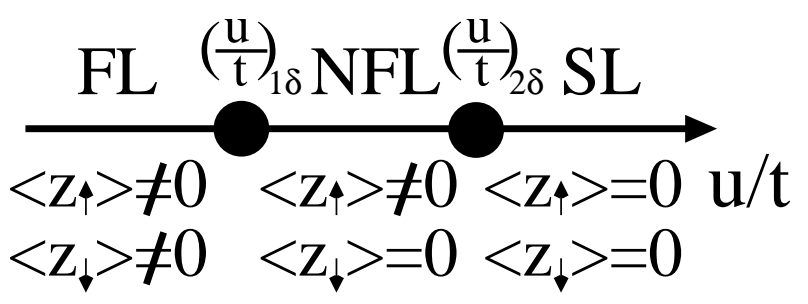

FIG. 1: A phase diagram in the SU(2) slave-rotor theory of the Hubbard model away from half filling

$u_{c} / D$ for the Mott critical point increases as hole concentration becomes larger, consistent with our expectation. Remember that this Mott critical point is defined by the condensation of the $z_{i \uparrow}$ bosons while the $z_{i \downarrow}$ bosons are gapped owing to $-\mu_{c}-\varphi_{0 c}>0$. We identify this Mott critical point with $(u / t)_{2 \delta}$.

There is another critical point associated with the condensation of the $z_{i \downarrow}$ bosons with $\left\langle z_{i \uparrow}\right\rangle \neq 0$. It is clear that both the $z_{i \uparrow}$ and $z_{i \downarrow}$ bosons get condensed when $\lambda_{c}+E_{c} \epsilon_{-}=0\left(-\mu_{c}+\varphi_{0 c}=0\right)$ and $\lambda_{c}+E_{c} \epsilon_{+}=0$ $\left(-\mu_{c}-\varphi_{0 c}=0\right)$ are satisfied, i.e., $\mu_{c}=0$ and $\varphi_{0 c}=0$. Then, we obtain $\lambda_{c}=(1-\delta) D E_{c}$ from the last equation in Eq. (32). Inserting this expression with $E_{c}=1 / 2$ into the fourth equation in Eq. (32), we find the second quantum critical point

$$
\frac{u_{c}}{D}=\frac{1}{4(2-\delta)}
$$

The actual values are $u_{c} / D \approx 0.127$ at $\delta=0.010, u_{c} / D \approx$ 0.128 at $\delta=0.050$, and $u_{c} / D \approx 0.132$ at $\delta=0.100$. At half filling Eq. (34) recovers the Mott critical point $u_{c} / D=0.125$ of the $\mathrm{U}(1)$ slave-rotor theory exactly. We define this critical value as $(u / t)_{1 \delta}$. Note that $(u / t)_{1 \delta}<$ $(u / t)_{2 \delta}$ is satisfied as expected.

In summary, there is an intermediate region $(u / t)_{1 \delta}<$ $u / t<(u / t)_{2 \delta}$ away from half filling where only the $z_{i \uparrow}$ bosons are condensed, i.e., $\left\langle z_{i \uparrow}\right\rangle \neq 0$ and $\left\langle z_{i \downarrow}\right\rangle=0$. The phase in $u / t<(u / t)_{1 \delta}$ is characterized by $\left\langle z_{i \uparrow}\right\rangle \neq 0$ and $\left\langle z_{i \downarrow}\right\rangle \neq 0$, identified with the Fermi liquid metal while the phase in $u / t>(u / t)_{2 \delta}$ is given by $\left\langle z_{i \uparrow}\right\rangle=0$ and $\left\langle z_{i \downarrow}\right\rangle=0$, named as the spin liquid Mott insulator. We emphasize again that the presence of the intermediate phase in $(u / t)_{1 \delta}<u / t<(u / t)_{2 \delta}$ results from the pseudospin-dependent chemical potential $\sigma \varphi_{0}$ due to hole doping, causing the pseudospin $\mathrm{SU}(2)$ symmetry breaking. The mean-field phase diagram is summarized in Fig. 1, where "FL", "NFL", and "SL" represent Fermi liquid, non-Fermi liquid, and spin liquid, respectively. Notice that the intermediate phase is identified with the nonFermi liquid metal in the phase diagram Fig. 1. In the following we investigate the nature of this intermediate phase named as the non-Fermi liquid metal.

One cautious person may suspect that the intermediate phase is an artifact of gauge degrees of freedom. In other words, performing gauge rotations to this ground state results in the same phase as the Fermi liquid state. How- ever, this guess is partially correct only. It is important to discriminate the pseudospin $\mathrm{SU}(2)$ symmetry from the $\mathrm{SU}(2)$ gauge symmetry. As discussed before, hole doping causes the easy-axis anisotropy, breaking the SU(2) pseudospin symmetry explicitly. However, the $\mathrm{SU}(2)$ gauge symmetry cannot be broken.[14] Unfortunately, the intermediate phase breaks the $\mathrm{SU}(2)$ gauge symmetry. Is this phase a gauge artifact? Not necessarily. This situation is exactly the same as the characterization of superconductivity. Usually, we characterize superconductivity as the condensed phase of Higgs bosons (Cooper pairs). This identification necessarily breaks the $\mathrm{U}(1)$ gauge symmetry, thus cannot be right in a rigorous manner. In this respect there is no local order parameter to break the $\mathrm{U}(1)$ gauge symmetry. However, we should admit that this characterization is useful. The way to understand this identification is to break the gauge symmetry explicitly, fixing one gauge. Since the gauge symmetry is explicitly broken via gauge fixing, one can define the local order parameter such as Cooper pair bosons. Now we can understand the intermediate phase in the same way. Consider gauge fixing first. Then, measure the condensation of $\mathrm{SU}(2)$ rotor bosons. This is a physically appealing way to discriminate various phases. In the following section we show that the intermediate state is indeed different from the Fermi liquid phase owing to the presence of incoherent fermion pairs.

\section{NATURE OF THE INTERMEDIATE PHASE}

To reveal the nature of the intermediate phase, it is necessary to find an effective Lagrangian. Low energy boson excitations are considered to be

$$
U_{i}=\left(\begin{array}{cc}
z_{i \uparrow} & -z_{i \downarrow}^{\dagger} \\
z_{i \downarrow} & z_{i \uparrow}^{\dagger}
\end{array}\right) \approx\left(\begin{array}{cc}
\sqrt{x} & -\sqrt{1-x} e^{-i \theta_{i}} \\
\sqrt{1-x} e^{i \theta_{i}} & \sqrt{x}
\end{array}\right)
$$

for this ground state. Here $x=\left|\left\langle z_{i \uparrow}\right\rangle\right|^{2}$ is the condensation amplitude, not determined self-consistently in the previous saddle-point analysis owing to complexity. $\theta_{i}$ is a dynamic variable to guarantee partial freezing of charge fluctuations, i.e., $\left\langle z_{i \downarrow}\right\rangle=\sqrt{1-x}\left\langle e^{i \theta_{i}}\right\rangle=0$. The ansatz of Eq. (35) satisfies the uni-modular constraint $\sum_{\sigma}\left|z_{i \sigma}\right|^{2}=1$. Since the objective of this section is to find a physical picture for this intermediate phase, we do not try to determine the condensation amplitude $x$, and just assume its presence.

The above ansatz for the $\mathrm{SU}(2)$ matrix field leads the 
time-fluctuation term of the boson sector to be

$$
\begin{aligned}
& \frac{1}{4 u} \sum_{i} \operatorname{tr}\left(-i U_{i} \partial_{\tau} U_{i}^{\dagger}+\vec{\Omega}_{i} \cdot \vec{\tau}-i \varphi_{0} \tau_{3}+i \mu U_{i} \tau_{3} U_{i}^{\dagger}\right)^{2} \\
& =\frac{1}{2 u} \sum_{i}\left[(1-x)\left(\partial_{\tau} \theta_{i}+\Omega_{z i}-i \varphi_{0}-i \mu\right)^{2}\right. \\
& -2 \sqrt{x(1-x)}\left(\Omega_{x i} \cos \theta_{i}+\Omega_{y i} \sin \theta_{i}\right)\left(\partial_{\tau} \theta_{i}-2 i \mu\right) \\
& \left.+2\left(\Omega_{x i}^{2}+\Omega_{y i}^{2}\right)+2 x\left(\Omega_{z i}-i \varphi_{0}+i \mu\right)^{2}\right]
\end{aligned}
$$

where $\vec{\Omega}_{i}$ represents the time component of the $\mathrm{SU}(2)$ gauge field around its zero mean-value. The $2 x\left(\Omega_{z i}-\right.$ $\left.i \varphi_{0}+i \mu\right)^{2}$ term allows us to replace $\Omega_{z i}$ with $i \varphi_{0}-i \mu$ in the low energy limit. This corresponds to the AndersonHiggs mechanism, as will be also seen in the boson hopping term. Inserting $\Omega_{z i}=i \varphi_{0}-i \mu$ into Eq. (36), we obtain the following expression for the right-hand-side (RHS) in Eq. (36)

$$
\begin{aligned}
& \text { RHS } \\
& =\frac{1-x}{2 u} \sum_{i}\left(\partial_{\tau} \theta_{i}-2 i \mu-\sqrt{\frac{x}{1-x}}\left[\Omega_{x i} \cos \theta_{i}+\Omega_{y i} \sin \theta_{i}\right]\right)^{2} \\
& +\frac{1}{u} \sum_{i}\left(\Omega_{x i}^{2}+\Omega_{y i}^{2}-\frac{x}{2}\left[\Omega_{x i} \cos \theta_{i}+\Omega_{y i} \sin \theta_{i}\right]^{2}\right) .
\end{aligned}
$$

One can see $\Omega_{x i}$ and $\Omega_{y i}$ fluctuations gapped from

$$
\begin{aligned}
& \frac{1}{u} \sum_{i}\left(\Omega_{x i}^{2}+\Omega_{y i}^{2}-\frac{x}{2}\left[\Omega_{x i} \cos \theta_{i}+\Omega_{y i} \sin \theta_{i}\right]^{2}\right) \\
& =\frac{1}{u} \sum_{i}\left(\left[1-x \cos ^{2} \theta_{i}\right] \Omega_{x i}^{2}+\left[1-x \sin ^{2} \theta_{i}\right] \Omega_{y i}^{2}\right. \\
& \left.+\frac{x}{2}\left[\Omega_{x i} \cos \theta_{i}-\Omega_{y i} \sin \theta_{i}\right]^{2}\right),
\end{aligned}
$$

thus safely ignored in the low energy limit. As a result, we find the low energy part for the time-fluctuation term

$$
\begin{aligned}
& \frac{1}{4 u} \sum_{i} \operatorname{tr}\left(-i U_{i} \partial_{\tau} U_{i}^{\dagger}+\vec{\Omega}_{i} \cdot \vec{\tau}-i \varphi_{0} \tau_{3}+i \mu U_{i} \tau_{3} U_{i}^{\dagger}\right)^{2} \\
& \approx \frac{1-x}{2 u} \sum_{i}\left(\partial_{\tau} \theta_{i}-2 i \mu\right)^{2} .
\end{aligned}
$$

To obtain the low energy sector for the boson hopping term, we use the following representation for the $\mathrm{SU}(2)$ gauge matrix $W_{i j}$

$$
W_{i j}=e^{-i \vec{a}_{i j} \cdot \vec{\tau}} \equiv\left(\begin{array}{cc}
X_{i j \uparrow} & -X_{i j \downarrow}^{\dagger} \\
X_{i j \downarrow} & X_{i j \uparrow}^{\dagger}
\end{array}\right)
$$

with the constraint $\left|X_{i j \uparrow}\right|^{2}+\left|X_{i j \downarrow}\right|^{2}=1$. Then, the boson hopping term is written as

$$
\begin{aligned}
& -t E \sum_{i j} \operatorname{tr}\left(U_{j}^{\dagger} \tau_{3} W_{i j} U_{i} \tau_{3}\right) \\
& =-t E \sum_{i j}\left[x\left(X_{i j \uparrow}+X_{i j \uparrow}^{\dagger}\right)-(1-x)\left\{e^{-i \theta_{j}} X_{i j \uparrow}^{\dagger} e^{i \theta_{i}}\right.\right. \\
& \left.+e^{i \theta_{j}} X_{i j \uparrow} e^{-i \theta_{i}}\right\}-\sqrt{x(1-x)}\left\{X_{i j \downarrow}^{\dagger} e^{i \theta_{i}}+X_{i j \downarrow} e^{-i \theta_{i}}\right. \\
& \left.\left.+X_{i j \downarrow} e^{-i \theta_{j}}+X_{i j \downarrow}^{\dagger} e^{i \theta_{j}}\right\}\right] .
\end{aligned}
$$

The first term in the RHS makes the $X_{i j \uparrow}$ bosons condensed. This is nothing but the Anderson-Higgs mechanism because the condensation of the $z_{i \uparrow}$ bosons makes the gauge fluctuations in the $X_{i j \uparrow}$ fields gapped. This corresponds to the fact that the $\phi_{3 i}$ condensation causes gapped $a_{3 i j}$ excitations in the U(1) slave-rotor gauge theory. Considering the uni-modular constraint, we can set $X_{i j \uparrow}=\sqrt{y}$ and $X_{i j \downarrow}=\sqrt{1-y} e^{i a_{i j}}$ as $z_{i \uparrow}=\sqrt{x}$ and $z_{i \downarrow}=\sqrt{1-x} e^{i \theta_{i}}$ of Eq. (35), where $y$ is the condensation amplitude. Then, the boson hopping term is obtained to be

$$
\begin{aligned}
& -t E \sum_{i j} \operatorname{tr}\left(U_{j}^{\dagger} \tau_{3} W_{i j} U_{i} \tau_{3}\right) \\
& =-t E \sum_{i j}\left[2 x \sqrt{y}-2(1-x) \sqrt{y} \cos \left(\theta_{i}-\theta_{j}\right)\right. \\
& \left.-2 \sqrt{x(1-x)(1-y)}\left\{\cos \left(\theta_{i}-a_{i j}\right)+\cos \left(\theta_{j}-a_{i j}\right)\right\}\right] \\
& \rightarrow-t E \sum_{i j}\left[2 x \sqrt{y}-2(1-x) \sqrt{y} \cos \left(\theta_{i}-\theta_{j}\right)\right. \\
& \left.-2 \sqrt{x(1-x)(1-y)}\left\{\cos \left(\theta_{i}-\theta_{j}-\tilde{a}_{i j}\right)+\cos \tilde{a}_{i j}\right\}\right],
\end{aligned}
$$

where we shift the gauge field $a_{i j}$ as $\tilde{a}_{i j}=a_{i j}-\theta_{j}$ in the last line.

An important issue arises due to the $\cos \tilde{a}_{i j}$ potential term. One may claim that this term allows us to ignore the gauge fluctuations at low energies because it causes $\tilde{a}_{i j}$ excitations gapped. However, this problem does not seem to be so simple owing to the stiffness parameter in the cos term. When the condensation amplitudes $x$ and $y$ of the $z_{i \uparrow}$ and $X_{i j \uparrow}$ bosons respectively are close to 1 , the stiffness parameter of the cos potential becomes very small. This may cause gauge fluctuations gapless. One can argue that the cos potential would be always relevant in two space and one time dimensions $[(2+1) D]$ in the renormalization group sense. However, this claim can be applied to the conventional sine-Gordon model in $(2+1) D$.[15] The present problem is more complex since gauge fluctuations also couple to fermion excitations, resulting in screening and dissipation in the gauge dynamics.

First, we discuss the case when the $\cos \tilde{a}_{i j}$ term is relevant. Then, we shift $\tilde{a}_{i j}$ as $\tilde{a}_{i j}+\pi$ due to the minus sign of the cos potential, and set $\tilde{a}_{i j}+\pi=$ $a_{i j}-\theta_{j}+\pi=0$ owing to the relevance of the cos 
term. This is an important constraint for gauge fluctuations. Because we consider the intermediate meanfield phase characterized by $\left\langle z_{i \downarrow}\right\rangle=\sqrt{1-x}\left\langle e^{i \theta_{i}}\right\rangle=0$ and $\left\langle z_{i \uparrow}\right\rangle=\sqrt{x}$, gauge fluctuations cause $\left\langle e^{i a_{i j}}\right\rangle=0$ that results from the constraint $\left\langle e^{i a_{i j}}\right\rangle=\left\langle e^{i\left(\theta_{j}-\pi\right)}\right\rangle$. As a result, the $\mathrm{SU}(2)$ gauge matrix $W_{i j}$ is found to be $W_{i j} \approx\left(\begin{array}{cc}\sqrt{y} & \sqrt{1-y} e^{-i a_{i j}} \\ -\sqrt{1-y} e^{i a_{i j}} & \sqrt{y}\end{array}\right)$ with $\left\langle e^{i a_{i j}}\right\rangle=0$. Note that this $\mathrm{SU}(2)$ gauge matrix is consistent with the mean-field analysis in Eq. (28) since this expression is reduced to the unit matrix in the mean-field level owing to $\left\langle e^{i a_{i j}}\right\rangle=0$. We emphasize that the off-diagonal components of the gauge matrix can arise in the intermediate phase beyond the mean-field approximation, associated with fermion pairing.

Using this gauge matrix, we find the following expression for the fermion hopping term

$$
\begin{aligned}
& -t F \sum_{i j} \eta_{i}^{\dagger} W_{i j}^{\dagger} \tau_{3} \eta_{j}=-t F \sum_{i j}\left[\sqrt{y}\left(\eta_{i+}^{\dagger} \eta_{j+}+\eta_{j-}^{\dagger} \eta_{i-}\right)\right. \\
& \left.+\sqrt{1-y}\left(\eta_{i+}^{\dagger} e^{-i a_{i j}} \eta_{j-}^{\dagger}+\eta_{i-} e^{i a_{i j}} \eta_{j+}\right)\right] .
\end{aligned}
$$

The key feature in the fermion hopping term is that fermion pairing is dynamically generated beyond the mean-field approximation due to the off-diagonal components of the $\mathrm{SU}(2)$ gauge matrix although this fermion pairing vanishes in the mean-field fashion owing to $\left\langle e^{i a_{i j}}\right\rangle=0$. This fermion hopping term identifies the off-diagonal gauge fluctuations with phase fluctuations of fermion pairs. Since the $\eta_{i}$ fermion carries an electric charge owing to the condensation of the $z_{i \uparrow}$ bosons, we interpret these dynamically generated fermion pairs as preformed Cooper pairs owing to strong phase fluctuations of the fermion pairs. An interesting point is that the preformed pairs arise from the kinetic energy term instead of the potential term in the Hubbard model.

The low energy effective Lagrangian for this phase is found to be

$$
\begin{aligned}
& L_{\eta}=\sum_{i \sigma} \eta_{i \sigma}^{\dagger}\left(\partial_{\tau}-\mu\right) \eta_{i \sigma}-t F \sqrt{y} \sum_{\langle i j\rangle \sigma}\left(\eta_{i \sigma}^{\dagger} \eta_{j \sigma}+H . c .\right) \\
& -t F \sqrt{1-y} \sum_{\langle i j\rangle} e^{-i a_{i j}}\left(\eta_{i+}^{\dagger} \eta_{j-}^{\dagger}-\eta_{i-}^{\dagger} \eta_{j+}^{\dagger}\right)-H . c . \\
& L_{\theta}=\frac{1-x}{2 u} \sum_{i}\left(\partial_{\tau} \theta_{i}-2 i \mu\right)^{2} \\
& -2 t E \sum_{\langle i j\rangle}[\sqrt{x(1-x)(1-y)}-(1-x) \sqrt{y}] \cos \left(\theta_{i}-\theta_{j}\right),
\end{aligned}
$$

where we use $\Omega_{z i}=i \varphi_{0}-i \mu$ in the time-fluctuation term for the $\eta_{i}$ fermions, obtained in the boson hopping term. Remember the gauge constraint $\tilde{a}_{i j}+\pi=a_{i j}-\theta_{j}+\pi=0$. One can suspect this derivation because the pairing symmetry is $s$-wave instead of $d-$ wave. In fact, the pairing symmetry depends on the sign of the gauge matrix $W_{i j}$. Thus, one can obtain the $d$-wave fermion pairing from the kinetic energy term considering $X_{i j \downarrow}=$ $-\sqrt{1-y} \varsigma_{i j} e^{i a_{i j}}$, where $\varsigma_{i j}$ is + when $j=i \pm \hat{x}$, and when $j=i \pm \hat{y}$. In this case the low energy effective Lagrangian becomes

$$
\begin{aligned}
& L_{\eta}=\sum_{i \sigma} \eta_{i \sigma}^{\dagger}\left(\partial_{\tau}-\mu\right) \eta_{i \sigma}-t F \sqrt{y} \sum_{\langle i j\rangle \sigma}\left(\eta_{i \sigma}^{\dagger} \eta_{j \sigma}+H . c .\right) \\
& -t F \sqrt{1-y} \sum_{\langle i j\rangle} \varsigma_{i j} e^{-i a_{i j}}\left(\eta_{i+}^{\dagger} \eta_{j-}^{\dagger}-\eta_{i-}^{\dagger} \eta_{j+}^{\dagger}\right)-H . c . \\
& L_{\theta}=\frac{1-x}{2 u} \sum_{i}\left(\partial_{\tau} \theta_{i}-2 i \mu\right)^{2} \\
& -2 t E \sum_{\langle i j\rangle}\left[\varsigma_{i j} \sqrt{x(1-x)(1-y)}-(1-x) \sqrt{y}\right] \cos \left(\theta_{i}-\theta_{j}\right) .
\end{aligned}
$$

It is not easy to determine which pairing symmetry will appear without more sophisticated analysis. However, one can see that if he compares the boson Lagrangian in Eq. (43) with that in Eq. (42), the stiffness parameter in Eq. (43) is larger than that in Eq. (42). In the case of $d$-wave pairing we find $\rho_{x}=t E \mid \sqrt{x(1-x)(1-y)}+$ $(1-x) \sqrt{y} \mid$ and $\rho_{y}=t E|\sqrt{x(1-x)(1-y)}-(1-x) \sqrt{y}|$, where $\rho_{x(y)}$ is the stiffness parameter in the $x(y)$ direction. On the other hand, we obtain $\rho_{x}=\rho_{y}=$ $t E|\sqrt{x(1-x)(1-y)}-(1-x) \sqrt{y}|$ in the case of $s$-wave pairing. Because the stiffness parameter in the $d-$ wave case is larger than that in the $s$-wave case, we expect that $d-$ wave pairing may be more favorable than $s-$ wave pairing.

Note that if the condensation amplitude $x$ is close to 1 , the assumption of $\left\langle z_{i \downarrow}\right\rangle=\sqrt{1-x}\left\langle e^{i \theta_{i}}\right\rangle=0$ is consistent with the small stiffness parameter in Eq. (43). In this case the resulting fermion Lagrangian becomes with the electromagnetic vector potential $A_{i j}$

$$
\begin{aligned}
& L_{e f f}=\sum_{i \sigma} \eta_{i \sigma}^{\dagger}\left(\partial_{\tau}-\mu\right) \eta_{i \sigma} \\
& -t F \sqrt{y} \sum_{\langle i j\rangle \sigma}\left(\eta_{i \sigma}^{\dagger} e^{i A_{i j}} \eta_{j \sigma}+H . c .\right) \\
& -t F \sqrt{1-y} \sum_{\langle i j\rangle} \varsigma_{i j} e^{-i a_{i j}}\left(\eta_{i+}^{\dagger} \eta_{j-}^{\dagger}-\eta_{i-}^{\dagger} \eta_{j+}^{\dagger}\right)-H . c . \\
& -\frac{1}{g^{2}} \sum_{\square} \cos (\partial \times a),
\end{aligned}
$$

where the last gauge action is introduced to impose the condition $\left\langle e^{-i a_{i j}}\right\rangle=0$ with an internal gauge charge $g$ of the $\eta_{i}$ fermion.

Eq. (44) is the main result of this section. Based on this effective Lagrangian for the intermediate phase, we discuss its physical implication. If quantum corrections due to gauge fluctuations $a_{i j}$ are ignored as the meanfield approximation, the effective fermion Lagrangian is 
reduced to

$$
\begin{aligned}
& L_{e f f}=\sum_{i \sigma} \eta_{i \sigma}^{\dagger}\left(\partial_{\tau}-\mu\right) \eta_{i \sigma} \\
& -t F \sqrt{y} \sum_{\langle i j\rangle \sigma}\left(\eta_{i \sigma}^{\dagger} e^{i A_{i j}} \eta_{j \sigma}+H . c .\right) .
\end{aligned}
$$

This seems to coincide with the Fermi liquid metal. Allowing the density-rotor variable only as the U(1) slaverotor representation, the Fermi liquid metal appears when the charge-rotor bosons are condensed.[4] Actually, the charge-rotor condensation makes the fermion field $\eta_{i}$ couple to the electromagnetic field $A_{i j}$. However, the presence of the pair-rotor variable is expected to alter this physical picture since the $\eta_{i \sigma}$ fermions are not electrons but fractionalized ones due to gapped pair-rotor excitations. To see the existence of coherent electron excitations, we consider the electron spectral function given by the convolution integral between the $\eta_{i \sigma}$ and $z_{i \sigma}$ propagators

$$
\begin{aligned}
& G_{e l \uparrow \uparrow}\left(i j, \tau \tau^{\prime}\right)=\left\langle T_{\tau}\left[c_{\uparrow i \tau} c_{\uparrow j \tau^{\prime}}^{\dagger}\right]\right\rangle \\
& =\left\langle T_{\tau}\left[U_{i \tau}^{\dagger} \eta_{i \tau} \eta_{j \tau^{\prime}}^{\dagger} U_{j \tau^{\prime}}\right]_{11}\right\rangle \approx x\left\langle T_{\tau}\left[\eta_{i \tau+} \eta_{j \tau^{\prime}+}^{\dagger}\right]\right\rangle \\
& +(1-x)\left\langle T_{\tau}\left[e^{-i \theta_{i \tau}} e^{i \theta_{j \tau^{\prime}}}\right]\right\rangle\left\langle T_{\tau}\left[\eta_{i \tau-}^{\dagger} \eta_{j \tau^{\prime}-}\right]\right\rangle .
\end{aligned}
$$

There exist coherent electron excitations in the meanfield fashion, resulting from the condensation of the $z_{i \uparrow}$ bosons. In this respect one can say that the intermediate phase corresponds to the Fermi liquid state. But, it should be noted that this result obtains in the saddlepoint approximation ignoring gauge fluctuations $a_{i j}$. The presence of gauge fluctuations alters this picture completely. Notice how the Fermi liquid renormalizes as a result of strong interactions. When $x=1$, this renormalized Fermi liquid metal becomes identical with the Fermi gas. Away from $x=1$ we find the quasiparticle weight $Z_{e l} \sim x$, reduced by the presence of gapped pairrotor excitations, where the remaining portion $1-x$ is transferred into incoherent backgrounds.

Strong gauge fluctuations corresponding to phase fluctuations of fermion pairs should be allowed. Then, we expect that this phase can be identified with a nonFermi liquid metal owing to pairing fluctuations. The presence of pairing-phase fluctuations is the hall mark to discriminate this phase from the Fermi liquid metal, represented as $\mathrm{U}(1)$ pair-gauge excitations. It is well known that the presence of long-range gauge interactions can result in non-Fermi liquid physics.[16] When gauge fluctuations are minimally coupled to gapless fermions, thus screening of gauge fluctuations occurs via particle-hole excitations, the effective $\mathrm{U}(1)$ gauge theory is characterized by the dynamical critical exponent $z=3$. In this case the imaginary part of the fermion self-energy is given by $\omega^{2 / 3}$ at the Fermi surface, implying that its real part also has the same frequency dependence via the Kramer's Kronig relation, thus giving rise to a nonFermi liquid behavior.[16] The $\gamma$ coefficient of the specific heat is proportional to $-\ln T$ in three spatial dimensions, and $T^{-1 / 3}$ in two dimensions. [17] The dc conductivity is proportional to $T^{-5 / 3}$ in three dimensions and $T^{-4 / 3}$ in two dimensions.[18] However, this non-Fermi liquid physics cannot be applied to the present case because screening of gauge fluctuations arises in particle-particle excitations instead of the particle-hole channel. Unfortunately, we don't know the role of pair-gauge fluctuations in the non-Fermi liquid physics at present. This should be investigated near future.

On the other hand, if the $\theta_{i}$ fluctuations are suppressed, i.e., $\left\langle z_{i \downarrow}\right\rangle=\sqrt{1-x}\left\langle e^{i \theta_{i}}\right\rangle \neq 0$, the corresponding gauge fluctuations would be also suppressed owing to the gauge constraint. In this case the resulting effective Lagrangian is obtained to be

$$
\begin{aligned}
& L_{e f f}=\sum_{i \sigma} \eta_{i \sigma}^{\dagger}\left(\partial_{\tau}-\mu\right) \eta_{i \sigma} \\
& -t F \sqrt{y} \sum_{\langle i j\rangle \sigma}\left(\eta_{i \sigma}^{\dagger} e^{i A_{i j}} \eta_{j \sigma}+H . c .\right) \\
& -t F \sqrt{1-y} \sum_{\langle i j\rangle} \varsigma_{i j}\left(\eta_{i+}^{\dagger} \eta_{j-}^{\dagger}-\eta_{i-}^{\dagger} \eta_{j+}^{\dagger}\right)-H . c .
\end{aligned}
$$

This is nothing but the effective Lagrangian for the $d$-wave BCS superconductivity. Thus, the $d$-wave BCS superconductivity can result from softening of pairing excitations in the SU(2) slave-rotor gauge theory. The transition nature from the anomalous metal with incoherent pairing to the $d$-wave superconductor is beyond the scope of this paper.

The $\mathrm{SU}(2)$ slave-rotor gauge theory can find the Fermi liquid, superconductivity, non-Fermi liquid, and spin liquid in principle. The Fermi liquid is described by $0<$ $\left\langle z_{i \uparrow}\right\rangle=\sqrt{x} \leq 1$ with any $\left\langle z_{i \downarrow}\right\rangle$ and $\left\langle X_{i j \uparrow}\right\rangle=\sqrt{y}=1$, $X_{i j \downarrow}=0\left(W_{i j}=I\right)$. The first condition results in the coherent electron-quasiparticle weight given by Eq. (46). The second condition indicates the absence of both diagonal and off-diagonal fluctuations of the SU(2) gauge matrix fields, where the absence of diagonal gauge fluctuations originates from the Anderson-Higgs mechanism due to the $z_{i \uparrow}$ condensation while the complete suppression of off-diagonal gauge fluctuations should be determined by the self-consistent mean-field analysis, not performed in this paper. In our mean-field analysis Eq. (28) both the diagonal and off-diagonal bosons are condensed, i.e. $\left\langle z_{i \sigma}\right\rangle \neq 0$, and the complete suppression of the offdiagonal components in the $\mathrm{SU}(2)$ gauge matrix is assumed, i.e., $W_{i j}=I$.

Superconductivity appears when $0<\left\langle z_{i \uparrow}\right\rangle=\sqrt{x}<1$, $0<\left\langle z_{i \downarrow}\right\rangle=\sqrt{1-x}<1$ and $0 \leq\left\langle X_{i j \uparrow}\right\rangle=\sqrt{y}<1$, $0<\left\langle X_{i j \downarrow}\right\rangle=\sqrt{1-y} \leq 1$. The presence of the offdiagonal $z_{i \downarrow}$ condensation is necessary for the off-diagonal gauge bosons $\left(X_{i j \downarrow}\right)$ to be condensed, causing coherent Cooper pairs for superconductivity given by Eq. (47). Unfortunately, we could not see superconductivity in our mean-field analysis because the off-diagonal components in the SU(2) gauge matrix are not allowed from the start. It is necessary to perform more sophisticated mean-field analysis allowing the off-diagonal gauge components. 
The anomalous metal arises when $0<\left\langle z_{i \uparrow}\right\rangle=\sqrt{x}<1$, $\left\langle z_{i \downarrow}\right\rangle=\sqrt{1-x}\left\langle e^{i \theta_{i}}\right\rangle=0$ and $0 \leq\left\langle X_{i j \uparrow}\right\rangle=\sqrt{y}<1$, $\left\langle X_{i j \downarrow}\right\rangle=\sqrt{1-y}\left\langle e^{i a_{i j}}\right\rangle=0$. One important thing is that we cannot see the non-Fermi liquid phase in the meanfield approximation ignoring gauge fluctuations. To obtain the anomalous metallic phase, off-diagonal components should be allowed in the $\mathrm{SU}(2)$ gauge matrix $W_{i j}$, meaning that the amplitudes of the off-diagonal components should be nonzero, and the expectation values of the off-diagonal components should be zero due to their strong gauge fluctuations, not captured in the present saddle-point analysis. What we have shown in our meanfield analysis is that the intermediate phase characterized by partial freezing of charge fluctuations, $\left\langle z_{i \uparrow}\right\rangle \neq 0$ and $\left\langle z_{i \downarrow}\right\rangle=0$, can appear near the Mott-Hubbard critical point. We see that this intermediate phase exhibits the Fermi liquid physics in the saddle-point approximation. However, the pair-gauge fluctuations (the off-diagonal gauge components) should be allowed, modifying the Fermi liquid physics completely. $\left\langle z_{i \downarrow}\right\rangle=\sqrt{1-x}\left\langle e^{i \theta_{i}}\right\rangle=$ 0 causes $\left\langle X_{i j \downarrow}\right\rangle=\sqrt{1-y}\left\langle e^{i a_{i j}}\right\rangle=0$ self-consistently. As a result, this intermediate phase is described by Eq. (44) with incoherent pairing fluctuations.

The spin liquid Mott insulator is given by $\left\langle z_{i \uparrow}\right\rangle=0$, $\left\langle z_{i \downarrow}\right\rangle=0$ and $\left\langle X_{i j \uparrow}\right\rangle=0,\left\langle X_{i j \downarrow}\right\rangle=0$. This is consistent with the mean-field analysis Eq. (28) except $X_{i j \downarrow}=0$, implying that this phase is identified with the $\mathrm{U}(1)$ spin liquid Mott insulator in the context of our mean-field analysis. On the other hand, if one admits fluctuations of the $\mathrm{SU}(2)$ gauge matrix fields, this insulating phase is interpreted as the $\mathrm{SU}(2)$ spin liquid Mott insulator due to $\mathrm{SU}(2)$ gauge fluctuations. In the case of $\mathrm{U}(1)$ gauge fluctuations it was claimed that the $\mathrm{U}(1)$ spin liquid Mott insulator can be stable against confinement resulting from instanton excitations when there exist sufficiently large flavors of gapless fermion excitations.[19, 20] However, in the case of $\mathrm{SU}(2)$ gauge fluctuations the $\mathrm{SU}(2)$ spin liquid Mott insulator would be unstable against confinement due to the $\mathrm{SU}(2)$ gauge fluctuations[21, 22] although there are no reliable calculations for this confinement problem owing to its complexity. If the confinement is realized at low energies, the $\mathrm{SU}(2)$ spin liquid is expected to turn into an antiferromagnetic insulator due to particle-hole confinement. An interesting possibility can arise that deconfined fermion excitations usually called spinons may appear at high energies owing to the asymptotic freedom[21] of the $\mathrm{SU}(2)$ gauge theory. This may explain the unidentified broad spin spectrum in the antiferromagnetic phase of high $\mathrm{T}_{c}$ cuprates, observed at high energies in the inelastic neutron scattering measurements.[23]

So far, we discussed the case when the $\cos \tilde{a}_{i j}$ term is relevant. Now we consider the case when the $\cos \tilde{a}_{i j}$ term is irrelevant. Shifting $\tilde{a}_{i j}$ as $\tilde{a}_{i j}+\pi$ due to the minus sign of the cos potential, we obtain the $\mathrm{SU}(2)$ gauge $\operatorname{matrix} W_{i j} \approx\left(\begin{array}{cc}\sqrt{y} & \sqrt{1-y} e^{-i a_{i j}} \\ -\sqrt{1-y} e^{i a_{i j}} & \sqrt{y}\end{array}\right)$. An important difference from the previous case is that there is no gauge constraint for $a_{i j}$ and $\theta_{j}$ because the $\cos \tilde{a}_{i j}$ term is assumed to be irrelevant. In this case $\tilde{a}_{i j}, a_{i j}$, and $\theta_{j}$ are all strongly fluctuating. The low energy effective Lagrangian is obtained to be

$$
\begin{aligned}
& L_{\eta}=\sum_{i \sigma} \eta_{i \sigma}^{\dagger}\left(\partial_{\tau}-\mu\right) \eta_{i \sigma}-t F \sqrt{y} \sum_{\langle i j\rangle \sigma}\left(\eta_{i \sigma}^{\dagger} \eta_{j \sigma}+H . c .\right) \\
& -t F \sqrt{1-y} \sum_{\langle i j\rangle} \varsigma_{i j} e^{-i a_{i j}}\left(\eta_{i+}^{\dagger} \eta_{j-}^{\dagger}-\eta_{i-}^{\dagger} \eta_{j+}^{\dagger}\right)-H . c . \\
& L_{\theta}=\frac{1-x}{2 u} \sum_{i}\left(\partial_{\tau} \theta_{i}-2 i \mu\right)^{2} \\
& -2 t E \sum_{i j}\left[\varsigma_{i j} \sqrt{x(1-x)(1-y)} \cos \left(\theta_{i}-\theta_{j}-\tilde{a}_{i j}\right)\right. \\
& \left.-(1-x) \sqrt{y} \cos \left(\theta_{i}-\theta_{j}\right)\right] .
\end{aligned}
$$

In the intermediate phase $\left\langle e^{i \theta_{i}}\right\rangle=0$ the fermion Lagrangian $L_{\eta}$ in Eq. (48) recovers Eq. (44), thus the anomalous metal is allowed even in this case. On the other hand, when the $z_{i \downarrow}$ bosons become condensed, the $\tilde{a}_{i j}$ fluctuations would be suppressed due to the Anderson-Higgs mechanism, thus $a_{i j}$ also, resulting in superconductivity described by Eq. (47). However, this conclusion is in contrast with the assumption that the $\cos \tilde{a}_{i j}$ term is irrelevant. Thus, to be self-consistent between the assumption and conclusion, the $\cos \tilde{a}_{i j}$ term should be relevant.

\section{DISCUSSION AND SUMMARY}

In this paper we try to answer to the question how to incorporate Mott physics (charge fluctuations) in BCStype superconductivity. Particulary, the objective is to construct an effective theory controlling pairing fluctuations in the BCS-like effective model. Since the U(1) slave-rotor gauge theory allows local density fluctuations only, this decomposition cannot control pairing excitations. Remember that there are two kinds of gauge fluctuations in the $\mathrm{U}(1)$ slave-rotor gauge theory with fermion pairing [Eq. (3)], one of which corresponds to phase fluctuations of fermion pairs. Although the $\mathrm{U}(1)$ charge-rotor variable governs dynamics of density-gauge fluctuations, such boson excitations to control pair-gauge fluctuations do not exist in the effective U(1) gauge theory [Eq. (3)]. In this paper we find the missing collective charge fluctuations represented as the pair-rotor variable in the $\mathrm{SU}(2)$ slave-rotor gauge theory. The core of the $\mathrm{SU}(2)$ slave-rotor gauge theory is that two kinds of collective boson excitations are introduced to control two kinds of gauge fluctuations. One of the boson excitations corresponds to the density-rotor variable of the $\mathrm{U}(1)$ slave-rotor representation, and the other is associated with on-site pairing fluctuations, called the pairrotor variable. One of the gauge fluctuations is interpreted as the density-gauge field of the $\mathrm{U}(1)$ slave-rotor representation, and the other is identified with phase degrees of freedom for fermion pairs, called the pair-gauge 
field. Although the $\mathrm{SU}(2)$ gauge structure is complex, we demonstrate that the density-rotor variable controls the density-gauge field while the pair-rotor variable governs the pair-gauge field. Since dynamics of collective charge fluctuations can be controlled via the local-interaction strength $u / t$, pairing fluctuations are naturally handled.

We perform the saddle-point analysis based on the $\mathrm{SU}(2)$ slave-rotor gauge theory. The crucial point in this analysis is that the $\mathrm{SU}(2)$ pseudospin symmetry is explicitly broken via hole doping, causing the easy-axis anisotropy. The easy-axis anisotropy of the SU(2) pseudospin order parameter is reflected as the pseudospindependent effective chemical potential for the $\mathrm{SU}(2)$ slave-rotor variable, allowing that only local density fluctuations are softened while local pairing fluctuations remain gapped in the intermediate parameter range away from half filling. Since density-rotor excitations are condensed, this phase exhibits the Fermi liquid physics in the saddle-point approximation corresponding to its nonzero quasiparticle weight proportional to the condensation amplitude of the density-rotor bosons. However, we see that this intermediate phase differs from the Fermi liquid state beyond the mean-field approximation allowing gauge fluctuations. Because pair-rotor excitations are gapped in this phase, their corresponding pair-gauge excitations are strongly fluctuating. Remember that the pair-gauge fluctuations can be identified with phase fluctuations of fermion pairs. Thus, incoherent fermion pairing does exist in this phase. The presence of preformed fermion pairs discriminates this phase from the Fermi liquid state completely [Eq. (44)].

It is valuable to discuss intuitively why local charge fluctuations can be partially frozen away from half filling. At half filling an on-site density fluctuation should induce an on-site pair excitation. This is the origin why the density- and pair-rotor bosons should be coherent or incoherent simultaneously. Away from half filling the on-site density fluctuation need not give rise to the onsite pair fluctuation because the density fluctuations can occur between $|0\rangle$ and $|1\rangle$ sites, but the on-site pair fluctuations should appear between $|1\rangle$ and $|1\rangle$ sites, where $|0\rangle$ is an empty site and $|1\rangle$ is one-electron site. As a result, away from half filling the density-rotor bosons can be condensed against the on-site Coulomb repulsion using the $|0\rangle$ and $|1\rangle$ sites while the pair-rotor bosons are gapped due to the on-site Coulomb repulsion in the intermediate parameter range.

Several important issues remain open. The $\mathrm{SU}(2)$ slave-rotor gauge theory opens the possibility of superconductivity in the Hubbard model. Introducing the
$\mathrm{SU}(2)$ slave-rotor matrix causes the $\mathrm{SU}(2)$ gauge matrix naturally. Since the SU(2) gauge matrix allows its offdiagonal components, off-diagonal fermion pairing can appear naturally in the kinetic energy term. Unfortunately, we did not perform a mean-field analysis to determine the amplitudes of the off-diagonal components in the $\mathrm{SU}(2)$ gauge matrix owing to its complex structure. More sophisticated self-consistent saddle-point analysis should be performed near future for superconductivity in the Hubbard model. Another problem is how to understand the spin liquid Mott insulator away from half filling. Although the slave-rotor gauge theory admits the presence of the Mott insulator away from half filling owing to its special structure allowing double occupancy, more extensive numerical simulations should be performed for the Hubbard model. To understand the nature of the non-Fermi liquid phase with preformed pairs such as transport and thermodynamics, it is indispensable to find how to treat pair-gauge fluctuations.

The stability of the non-Fermi liquid metal with incoherent fermion pairing against disorder is an interesting open question for this phase to be a genuine metallic phase in two dimensions. The present author investigated the role of disorder in the two dimensional fermion system with long-range gauge interactions, where gauge fluctuations couple to charge currents instead of pair currents as the present case. [6, 24] Long range interactions are shown to make the fermion system stable against weak disorder even in two dimensions because the gauge interactions let the fermions lie in a critical phase, causing that the critical fermions feel their effective dimension higher than two owing to their anomalous critical exponents. Remember that there exists the localization-delocalization transition in the weak disorder limit above two dimensions. Criticality can protect fermions from localization due to disorder in the weak disorder limit. $[6,24,25]$ However, the present case should be addressed more thoroughly because gauge fluctuations couple to pair currents instead of charge currents. This problem may be related with the anomalous metallic behavior in two dimensions. [26, 27]

K.-S. Kim would like to thank Dr. A. Tanaka for pointing out the possibility for the $\mathrm{SU}(2)$ slave-rotor gauge theory to describe the gossamer metal in Ref. [3]. K.S. Kim appreciates helpful discussions with Drs. Y.-B. Kim, H.-Y. Kee, J.-W. Lee, K. Park, J.-H. Han, and B. J. Yang. K.-S. Kim expresses thanks to Dr. A. Rosch and his group members, particulary Dr. Markus for illuminating discussions.
[1] P. A. Lee, N. Nagaosa, and X.-G. Wen, Rev. Mod. Phys. 78, 17 (2006).

[2] A. Paramekanti, M. Randeria, and N. Trivedi, Phys. Rev. Lett. 87, 217002 (2001); A. Paramekanti, M. Randeria, and N. Trivedi, Phys. Rev. B 70, 054504 (2004).
[3] B. A. Bernevig, R. B. Laughlin, and D. I. Santiago, Phys. Rev. Lett. 91, 147003 (2003); Bogdan A. Bernevig, George Chapline, Robert B. Laughlin, Zaira Nazario, and David I. Santiago, cond-mat/0312573.

[4] S. Florens and A. Georges, Phys. Rev. B 70, 035114 
(2004).

[5] S.-S. Lee and P. A. Lee, Phys. Rev. Lett. 95, 036403 (2005).

[6] Ki-Seok Kim, Phys. Rev. B 73, 235115 (2006).

[7] The present decomposition scheme looks somewhat similar to the slave-boson representation of the $t-J$ model. However, our decomposition scheme is nothing to do with the slave-boson representation because the slave-boson representation originates from how to solve the single occupancy constraint in the $t-J$ model while the present decomposition does not. It should be noted that this decomposition is not arbitrary since the collective mode $U_{i \sigma \sigma^{\prime}}$ is determined by its HS field, as will be discussed in the text.

[8] Ki-Seok Kim, Phys. Rev. Lett. 97, 136402 (2006).

[9] Ki-Seok Kim, Phys. Rev. B 74, 115122 (2006).

[10] T.-P. Choy and P. Phillips, Phys. Rev. Lett. 95, 196405 (2005).

[11] K. Haule, A. Rosch, J. Kroha, and P. Wolfle, Phys. Rev. B 68, 155119 (2003); M. M. Zemljic and P. Prelovsek, Phys. Rev. B 72, 075108 (2005).

[12] At half filling $(\delta=0)$ we obtain $\varphi_{0}=0$ and $\mu=$ $-\sqrt{2 u(\lambda-D E)}$, causing $\epsilon_{-}=-D$. As a result, we obtain the following equations for the mean-field parameters $E, F$, and $\lambda$ at half filling

$$
\begin{aligned}
& E=\frac{1}{2}, \quad F=\frac{1}{3(D E)^{2}}[(2 \lambda-D E) \sqrt{2 u(\lambda+D E)} \\
& -(2 \lambda+D E) \sqrt{2 u(\lambda-D E)}] \\
& 1=\frac{\sqrt{2 u(\lambda+D E)}-\sqrt{2 u(\lambda-D E)}}{D E} .
\end{aligned}
$$

Solving these algebraic equations, we find the analytic expressions for the mean-field parameters

$$
\begin{aligned}
\lambda & =2 u+\frac{D^{2}}{32 u}, \quad E=\frac{1}{2}, \\
F & =\frac{\left(64 u^{2}+D^{2}-8 u D\right)(8 u+D)-\left(64 u^{2}+D^{2}+8 u D\right) \mid 8 u-D}{48 D^{2} u} \\
& =\frac{1}{24} \frac{D}{u} \quad \text { for } \quad \frac{u}{D} \geq \frac{1}{8} \\
& =\frac{64}{3}\left(\frac{u}{D}\right)^{2} \quad \text { for } \quad \frac{u}{D}<\frac{1}{8}
\end{aligned}
$$

Condensation of the $\phi_{3 i}$ bosons occurs when their excitation gap closes, given by $\mu_{c}=0$ or $\lambda_{c}-D E_{c}=0$ that determines the Mott-Hubbard critical point $u_{c} / D=1 / 8$ in the mean-field approximation.
[13] One can derive the pair-rotor theory Eq. (24) in the canonical quantization method by decomposing the electron Hilbert space into the composite Hilbert space of $|\psi\rangle=|\eta\rangle \otimes\left|\Delta^{R}\right\rangle$ based on the composite field representation $\psi_{i}=e^{-i \phi_{1 i} \tau_{1}} \eta_{i}$, where $\Delta^{R}$ represents the density of an electron pair. This enlarged composite Hilbert space is reduced to the original electron one by the constraint $\Delta_{i}^{R}=\psi_{i}^{\dagger} \tau_{1} \psi_{i}$. This canonical quantization can be realized in the following path integral expression

$$
\begin{aligned}
& Z=\int D\left[\eta_{i}, \phi_{1 i}, \Phi_{i}^{R}, \Delta_{i}^{R}\right] \exp \left[-\int d \tau\left\{\sum_{i} \eta_{i}^{\dagger} \partial_{\tau} \eta_{i}\right.\right. \\
& -t \sum_{i j} \eta_{i}^{\dagger} e^{i \phi_{1 i} \tau_{1}} \tau_{3} e^{-i \phi_{1 j} \tau_{1}} \eta_{j} \\
& \left.\left.+\sum_{i}\left(u \Delta_{i}^{R 2}-i \Delta_{i}^{R} \partial_{\tau} \phi_{1 i}+i \Phi_{i}^{R}\left(\Delta_{i}^{R}-\psi_{i}^{\dagger} \tau_{1} \psi_{i}\right)\right)\right\}\right],
\end{aligned}
$$

resulting in the pair-rotor theory Eq. (24) after integrating out the $\Delta_{i}^{R}$ field, and performing the HS transformation for the hopping term. The term $-i \Delta_{i}^{R} \partial_{\tau} \phi_{1 i}$ leads us to identify the $e^{-i \phi_{1 i} \tau_{1}}$ operator with an annihilation operator of an electron pair.

[14] S. Elitzur, Phys. Rev. D 12, 3978 (1975).

[15] H. Kleinert, F. S. Nogueira, and A. Sudbo, Phys. Rev. Lett. 88, 232001 (2002); H. Kleinert, F. S. Nogueira, and A. Sudbo, Nucl. Phys. B 666, 361 (2003).

[16] I. Vekhter and A. V. Chubukov, Phys. Rev. Lett. 93 016405 (2004).

[17] Ki-Seok Kim and Mun Dae Kim, cond-mat/0608235, to be published in Phys. Rev. B.

[18] P. A. Lee and N. Nagaosa, Phys. Rev. B 46, 5621 (1992).

[19] M. Hermele, T. Senthil, M. P. A. Fisher, P. A. Lee, N. Nagaosa, and X.-G. Wen, Phys. Rev. B 70, 214437 (2004).

[20] Ki-Seok Kim, Phys. Rev. B 72, 245106 (2005).

[21] A. M. Polyakov, Gauge Fields and Strings, Chap. 4 (Harwood Academic Publishers, 1987).

22] E. Fradkin, S. H. Shenker, Phys. Rev. D 19, 3682 (1979).

[23] Ki-Seok Kim, Phys. Rev. B 72, 214401 (2005).

[24] Ki-Seok Kim, Phys. Rev. B 72, 014406 (2005); Phys. Rev. B 70, 140405(R) (2004).

[25] S. Chakravarty, L. Yin, and E. Abrahams, Phys. Rev. B 58, R559 (1998).

[26] E. Abrahams, S. V. Kravchenko, and M. P. Sarachik, Rev. Mod. Phys. 73, 251 (2001).

[27] A. Kapitulnik, N. Mason, S. A. Kivelson, and S. Chakravarty, Phys. Rev. B 63, 125322 (2001). 